\title{
Use-Dependent Alterations of Movement Representations in Primary Motor Cortex of Adult Squirrel Monkeys
}

\author{
R. J. Nudo, ${ }^{1}$ G. W. Milliken, ${ }^{1}$ W. M. Jenkins, ${ }^{2}$ and M. M. Merzenich ${ }^{2}$ \\ 1 Department of Neurobiology and Anatomy, University of Texas Medical School at Houston, Houston, Texas 77030, and \\ ${ }^{2}$ Coleman Laboratory and W. M. Keck Center for Integrative Neuroscience, Departments of Physiology and \\ Otolaryngology, University of California at San Francisco, San Francisco, California 94143
}

\begin{abstract}
This study was undertaken to document plastic changes in the functional topography of primary motor cortex (M1) that are generated in motor skill learning in the normal, intact primate. Intracortical microstimulation mapping techniques were used to derive detailed maps of the representation of movements in the distal forelimb zone of $\mathrm{M} 1$ of squirrel monkeys, before and after behavioral training on two different tasks that differentially encouraged specific sets of forelimb movements. After training on a small-object retrieval task, which required skilled use of the digits, their evoked-movement digit representations expanded, whereas their evoked-movement wrist/forearm representational zones contracted. These changes were progressive and reversible. In a second motor skill exercise, a monkey pronated and supinated the forearm in a key (eyebolt)-turning task. In this case, the representation of the forearm expanded, whereas the digit representational zones contracted. These results show that $M 1$ is alterable by use throughout the life of an
\end{abstract}

animal. These studies also revealed that after digit training there was an areal expansion of dual-response representations, that is, cortical sectors over which stimulation produced movements about two or more joints. Movement combinations that were used more frequently after training were selectively magnified in their cortical representations. This close correspondence between changes in behavioral performance and electrophysiologically defined motor representations indicates that a neurophysiological corrclate of a motor skill resides in M1 for at least several days after acquisition. The finding that cocontracting muscles in the behavior come to be represented together in the cortex argues that, as in sensory cortices, temporal correlations drive emergent changes in distributed motor cortex representations.

Key words: motor cortex; primates; squirrel monkey; motor learning; plasticity; hand
Numerous studies conducted primarily in somatosensory cortical areas over the past several years have revealed that representational maps (i.e., somatotopic maps of the body surface) are altered by manipulations of their sensory inputs (for reviews, see Merzenich et al., 1988; Kaas, 1991) (see also Kalaska and Pomeranz, 1979; Kelehan et al., 1981; Rasmusson, 1982; Kaas et al., 1983; Merzenich et al., 1983a,b, 1984; Rasmusson and Turnbull, 1983; Kelehan and Doetsch, 1984; Simons et al., 1984; Rasmusson el al., 1985; Wall and Kads, 1986; Calford and Tweedale, 1988, 1991; Wall, 1988; Welker et al., 1989; Recanzone et al., 1990). Each manipulation resulted in substantial alterations in functional topography of somatosensory cortical areas. Although plastic changes in the cerebral cortex have been known for some time to occur developmentally, these studies demonstrating reorganization in adult primates indicate that cortical maps are alterable throughout life.

Although these studies demonstrate that reorganization in somatosensory cortex can be induced by pathological disturbances, more recent experiments show that sensory cortex is selectively altered by the behavioral experiences of intact animals. For ex-

Received Aug. 9, 1995; revised Oct. 3, 1995; accepted Oct. 5, 1995.

This work was supported by: NIH Grants NS-27974 (R.J.N.), NS-09366 (G.W.M.), and NS-10414 (M.M.M.); the State of Texas Advanced Research Program (Grant 011618025); and the Whitehall Foundation (R.J.N.). We thank Rama Grenda, Lara Weisheit, Ramin Raiszedeh, and Tim Peterson for assisting in data collection.

Correspondence should be addressed to Randolph J. Nudo, Department of Neurobiology and Anatomy, University of Texas Medical School at Houston, Houston, TX 77030.

Copyright 01996 Society for Neuroscience $0270-6474 / 96 / 160785-23 \$ 05.00 / 0$ ample, in adult owl monkeys, with behaviorally controlled tactile stimulation of one or two digit tips, the sensory representation of the stimulated skin surface in area $3 b$ enlarged, and engaged skin surfaces were represented in correspondingly finer grain (Jenkins et al., 1990). Similarly, after a frequency discrimination task, the topographies of the representations of the trained hand were more spatially complex and changes in distributed responses were strongly correlated with behaviorally documented performance gains. These studies have revealed that the details of representation of the body surface in somatosensory cortical areas reflect the recent bchavioral experiences of the animal under study (Recanzone et al., 1992b).

Evidence is also accumulating that indicates that motor cortex is similarly remodeled throughout life. For example, experiments conducted by Donoghue and colleagues have shown that cortical motor representations can be rapidly altered by peripheral nerve lesions in both developing and adult rats (Donoghue and Sanes, 1987; Sanes et al., 1990). Also, our own studies have shown that repetitive electrical stimulation of motor cortex, even at levels well below movement thresholds, induces changes in movement rep resentations (Nudo et al., 1990) (see also Graham-Brown and Sherrington, 1912; Leyton and Sherrington, 1917). Local injection of bicuculline results in a rapid induction of changes in cortical motor topography in adult rats (Jacobs and Donoghue, 1991). Taken together, these studies indicate that the functional organization of motor cortex, like that of somatosensory cortex, is modifiable and selectively alterable throughout life.

If the functional organization of "movement representations" 
in the motor cortex can be altered by these peripheral and central manipulations, it is likely that, normally, experiential manipulations continually reshape the details of functional topography in the motor cortex, as in SI somatosensory cortical areas. The role of motor cortex in the acquisition of motor skills rarely has been addressed in this way. Modern studies of the role of motor cortex in motor control have used highly trained (usually overtrained), awake, behaving animals, and neurophysiological data have been derived in nearly every experiment only after the animal has reached performance criteria for the behavior (Evarts et al., 1983). Because somatosensory experiments indicate that training results in substantial moditication of cortical maps, important interactions between behavioral performance and cortical physiology could be occurring in the motor cortex before these neurophysiological studies are initiated.

In the present experiment in adult squirrel monkeys, we tracked the topographies of distal forelimb representations in the primary motor cortical field (M1 or area 4), before and after behavioral training for a task that required the skilled use of the hand digits. The results show that motor cortex, like somatosensory cortex, is dynamically altered by behavioral experience. Severalfold increases in the complexity and in the cortical areas dedicated to the representations of specific movements and movement combinations that are important for the learned motor skill were recorded in these trained monkeys. At the end of a training epoch, M1 representations clearly were adapted specifically to selectively represent key aspects of the newly acquired skill.

\section{MATERIALS AND METHODS}

Sixteen detailed electrophysiological mapping studies were conducted in six adult male squirrel monkeys (Saimiri boliviensis peruviensis). Monkeys were young adults between 2 and 5 years of age. Each was free of any obvious physical or neurological deficits, and each was judged to be healthy before and after behavioral training and electrophysiological mapping sessions.

The general procedures were as follows. First, limited hand preference testing was conducted to determine each animal's hand preference for retrieval of small food pellets (see below). Several weeks to montins later, an electrophysiological map of the distal forelimb representation was derived within $\mathrm{M} 1$ in the hemisphere contralateral to the preferred hand. Each animal was then randomly assigned to one of two groups: (1) a behavioral training group $(n=3)$ that underwent daily training on a pellet retrieval task ("digit-training task"); or (2) a control group $(n=3)$ that underwent no specific behavioral training. In each animal, after behavioral training (training group) or an equivalent period without training (control group), identical electrophysiological mapping procedures were repeated. Finally, after the two control procedures, one monkey in the control group underwent daily training at a key (eyebolt)turning lask ("foream-(raining lask") folluwed by a final electrophysiological mapping procedure.

\section{Hand preference testing procedure}

Hand preference testing was conducted using a modified "Klüver board" (Glees, 1961; Lawrence and Kuypers, 1968) following procedures described in detail previously (Nudo et al., 1992). This device consisted of a rectangular Plexiglas board, $24 \times 7.6 \times 1.8 \mathrm{~cm}^{3}$ thick, containing four food wells of different diameters: $25,19.5,13$, and $9.5 \mathrm{rmm}$, or wells 1,2 , 3 , and 4 , respectively. The vertical sides of each of these wells were $5 \mathrm{~mm}$ high; the bottom of the wells tapered at a $30^{\circ}$ angle to a central point.

Each animal was food-restricted for $18 \mathrm{hr}$ before the hand preference testing procedure was conducted. Hand use preference was determined for 100 trials over a period of 2-3 d. In these tests, the Klüver board was attached to the front of the animal's home cage, $\sim 12$ inches above the height of the cage floor. The animal was required to support itself with one hand while reaching through the cage bars to retrieve pellets with the other. Each trial was recorded on videotape for later analysis. A single, banana-flavored, $45 \mathrm{mg}$ food pellet (BioServe, Laurel, MD) was placed randomly into one of four wells, and the animal was allowed to retrieve it. If the pellet was not successfully retrieved within $1 \mathrm{~min}$, it was removed from the well and the next trial was begun. This time limit was imposed to minimize training effects. The videotape was reviewed later, and for each trial we recorded (1) the hand used on the initial reach for that trial, (2) the hand used on subsequent reaches, and (3) the hand used to dislodge or retrieve the pellet. Because these three measures were highly correlated, a "preference score" was derived for each animal that was equal to the average of the percentage of left-hand initial reaches and the percentage of left-hand retrievals for all wells.

\section{Behavioral training procedures}

Two types of behavioral training procedures were conducted: (1) a digit-training task using the same Klüver board used in hand preference testing; and (2) a forearm-training task using a rotatable eyebolt.

Digit-training task $(\mathrm{n}=3)$. Each animal was trained in two separate 30 min sessions/d. Monkeys were food-restricted for $18 \mathrm{hr}$ before each daily training session. All aspects of the training apparatus were identical to those used for hand preference testing. Training sessions consisted of alternating "priming periods" and "training periods." During each priming period, a single banana-flavored food pcllet was placed in the largest $(25 \mathrm{~mm})$ well until retrieval. Pellets were then placed in consecutively smaller wells until retrieval. This sequence was repeated for the duration of the priming period $(1 \mathrm{~min})$. During a training period, a single pellet was placed in the smallest $(9.5 \mathrm{~mm})$ well until retrieval. Upon retrieval, another pellet was placed in the same well. This procedure continued until another priming period began (i.e., for $4 \mathrm{~min}$ ) or until the end of the $30 \mathrm{~min}$ session. That is, during each priming period, retrieval from the smallest well was required on one of every four trials; during each training period, retrieval from the smallest well $(9.5 \mathrm{~mm})$ was required on every trial. Training continued for a minimum of $11 \mathrm{~d}$ and until the number of daily retricvals from the smallest $(9.5 \mathrm{~mm})$ wcll was $\geq 600$ pellets for 2 consecutive days.

In one animal, a titration procedure was required to encourage efficient retrieval in a second training phase. This procedure was begun 3 months after phase I training and the subsequent mapping procedure. The titration procedure was conducted as follows. A fifth well was introduced that was intermediate in size between the 13 - and 9.5-mm-diameter wells (wells 3 and 4, respectively). The diameter of this intermediate well was $11 \mathrm{~mm}$ (well 3.5). Titration training was conducted as in phase. I but, instead of conducting intensive training on well 4 , training was conducted initially on well 3 . That is, during each training period, retrieval from well $3(13 \mathrm{~mm})$ was required on every trial. Daily training continucd on wcll 3 until a criterion performance level was reached ( 2 of $3 \mathrm{~d},>600$ retrievals). The difficulty was then increased by introducing pellets into well 3.5 (11 $\mathrm{mm}$ ) on $10 \%$ of the trials until criterion performance was reached. This difficulty level was followed by $75 \%$ well $3 / 25 \%$ well 3.5 , then $50 \%$ well $3 / 50 \%$ well 3.5 , then $25 \%$ well $3 / 75 \%$ well 3.5 and, finally, $100 \%$ well 3.5 . The same procedure was used to progress eventually to criterion performance on well 4 . The final criterion on well $4(9.5 \mathrm{~mm})$ was 3 consecutive days with $>600$ retrievals.

Videotapes were examined later frame-by-frame to assess the frequency of movements and movement combinations used in the task at each phase of training. Samples consisting of selected trials on the smallest well (well 4) were examined. For the duration of each trial, we identified and coded each distal forelimb movement and movement combination used until retrieval of the pellet was achieved. Movement categories were identical to those used for describing movements evoked by intracortical microstimulation (see Electrophysiological mapping procedure below). Movement combinations were movements of multiple joints that occurred simultaneously: Discrete movement events were delineated by starts and stops in the animal's motion during any given trial. At each training phase, the frequency of each movement category and movement combination was tallied and expressed as a percentage of total movement events for that stage (see Table 3 ).

Forearm-training task $(\mathrm{n}=1)$. In a fourth animal, a behavioral training procedure was used that required repetitive cycles of supination and pronation of the forearm. 'The apparatus consisted of a rotatable eyebolt with an eye that was 1 inch in diameter. A metal rod was attached perpendicular to the axis of the eyebolt. As the eyebolt was rotated, the rod depressed one of two microswitches. The microswitches were arranged so that the maximum rotation of the eyebolt could be adjusted from a minimum of $20^{\circ}$ to a maximum of $45^{\circ}$. The task required that the animal make alternating clockwise and counterclockwise rotations of the eyebolt to receive a banana-flavored pellet delivered from an automatic dispenser. The entire apparatus was mounted on the animal's home cage and was available to the animal $24 \mathrm{hr} / \mathrm{d}$. 
The rotation angle initially was set at $20^{\circ}$, and the animal was rewarded on a $100 \%$ reinforcement schedule, that is, each time either microswitch was activated was whenever a supination or pronation of the forearm was made. When a criterion performance of 1000 pellets/d was reached, the animal was required to alternate activation of the two microswitches, that is, to alternate supination and pronation. When criterion performance on this task was reached, the rotation angle was increased in two steps, from $20^{\circ}$ to $32.5^{\circ}$ to $45^{\circ}$. Once criterion performance was reached at the $45^{\circ}$ rotation angle, a Plexiglas cylinder with an inside diameter of 1.5 inches was extended gradually over the eyebolt. This encouraged the use of one hand. The cylinder was extended in five steps to a distance just proximal to the elbow, to encourage forearm supinations and pronations while discouraging elbow flexion and elbow extension as well as lateral and medial rotation of the shoulder. When criterion performance was reached, an annulus was inserted into the end of the cylinder to further restrict movements of the proximal limb and shoulder. Once this task was performed proficiently, the schedule of reinforcement was reduced gradually to $5 \%$. Final criterion performance was achieved when the animal performed at least 10,000 forearm pronations and supinations on each of 2 consecutive days.

Control group $(\mathrm{n}=3)$. Three animals served as controls. In each animal, an electrophysiological mapping procedure was conducted. The animal was then placed back into its home cage. After a period of 1-2 months, a second, equally detailed electrophysiological mapping procedure was conducted.

\section{Electrophysiological mapping procedure}

Each animal was anesthetized initially with ketamine hydrochloride (20 $\mathrm{mg} / \mathrm{kg}$, i.m.). After tracheal intubation, the animal was administered halothane $(1-2 \%)$ and nitrous oxide $(75 \%)$ and placed in a stereotaxic frame. Core temperature was measured using a rectal probe; a homeothermic blanket maintained body temperature within $36.5-38.0^{\circ} \mathrm{C}$ throughout the experiment (typically 15-19 hr). Heart and respiration rates were monitored periodically. An indwelling cannula was inserted into the saphenous vein for delivery of intravenous fluids. Mannitol ( 8 $\mathrm{cc} / \mathrm{kg}, 250 \mathrm{mg} / \mathrm{cc}$ ) was administered slowly to reduce the likelihood of brain edema. Antibiotics (Crysticillin, $\sim 60,000 \mathrm{U}$ ) were administered before surgery and every $12 \mathrm{hr}$ thereafter during the procedure. Under sterile conditions, a limited craniotomy $(\sim 1.5 \mathrm{~cm}$ diameter) was made over M1. A small, plastic chamber was affixed with dental acrylic to the skull around the opening. After reflection of the dura, the chamber was filled with silicone oil (30k Centistoke, dimethylpolysiloxane, Dow 200 fluid).

The halothane and nitrous oxide were then withdrawn, and the animal was administered ketamine hydrochloride (diluted to $20 \mathrm{mg} / \mathrm{cc}$ with saline) intravenously at the rate of $15 \mathrm{mg} / \mathrm{kg} / \mathrm{hr}$. In addition, acepromazine (diluted to $0.1 \mathrm{mg} / \mathrm{cc}$ with saline) was administered as needed to control excessive muscle tone (typically $0.01 \mathrm{mg} / \mathrm{kg}$ per dose). The rate of continuous ketamine infusion was adjusted as needed to maintain a stable anesthetic state. Intracortical microstimulation (ICMS) mapping was conducted only during periods of stable anesthesia; it was halted during occasional periods of more shallow anesthesia marked by excessive muscle tone in forelimb muscles combined with rapid heart rate, or during occasional periods of deeper anesthesia marked by unusually high ICMS thresholds.

$\Lambda$ magnified photograph of the cortical surface vasculature was obtained using a video frame-grabber. Stimulating microelectrodes consisted of glass micropipettes with $15-20 \mu \mathrm{m}$ tip diameters. Micropipettes were $<100 \mu \mathrm{m} 2 \mathrm{~mm}$ from the tip and beveled to a sharp point. They were filled with $3.5 \mathrm{M} \mathrm{NaCl}$. Impedances ranged from $750 \mathrm{k} \Omega$ to $1 \mathrm{M} \Omega$. Microelectrodes were introduced on a fine grid pattern $(250 \mu \mathrm{m}$ interpenetration distances) sited with reference to the surface vasculature (see Fig. 3). At each electrode penetration site, conventional ICMS techniques were used to define evocable movements. Briefly, the micropipette was advanced perpendicular to the cortical surface to a depth of $1700-1800$ $\mu \mathrm{m}$ using a hydraulic microdrive. Our previous data, as well as previously published data by others (Strick and Preston, 1987a; Gould et al., 1986; Nudo et al., 1992), have shown that thresholds for evoking movements are the lowest in this layer 5 depth range. Significant dimpling of the cortical surface rarely occurred using beveled pipettes. However, when dimpling was observed the cortex was allowed to return to its original position before stimulation. The stimulus consisted of a 40 -msec-current train of thirteen $200-\mu$ sec-long monophasic cathodal pulses delivered at $350 \mathrm{pulses} / \mathrm{sec}$ from an electrically isolated, charge-balanced (capacitively coupled) stimulation circuit. Trains were delivered at a rate of $1 / \mathrm{sec}$.
Current was monitored by observing the voltage drop across a $10 \mathrm{k} \Omega$ resistor in series with the stimulation circuit.

In the derivation of all maps, animals were maintained in a standard prone position. The contralateral forelimb was supported below the elbow in the same slightly flexed position throughout each experiment. The forelimb was manipulated only when necessary to stabilize the wrist to distinguish between wrist extension and digit extension.

At each site, the movement evoked by stimulation at near-threshold current levels was determined by the following procedure. Current was increased gradually from zero until a response was just visible. When the evoked movement was defined, the current was decreased until the movement disappeared and then was increased again gradually to find the current level required for evoking the movement with at least $50 \%$ of the train bursts. This current was defined as the "threshold current." In each experiment, in a sample of $\sim 10 \%$ of electrode penetration sites, a second observer independently determined the responses and threshold currents. If a response was not evoked at $30 \mu \mathrm{A}$, stimulation was halted and the site was designated as "nonresponsive." A second movement was sometimes evoked reliably at or near threshold current levels. If a second movement was evoked consistently at $\leq 2 \mu \mathrm{A}$ above the threshold intensity, the movements were defined as unresolvable and that cortical location was designated as a "dual-response" site. At some sites, the movement and threshold evoked by stimulation were recorded at a range of depths from 1400 to $2000 \mu \mathrm{m}$. This test was performed to ensure that the electrode was perpendicular to the cortical surface and that the lowest possible current thresholds were obtained.

The territorial extent of the area 4 distal forelimb representation was explored as completely as possible by using the following criteria. Penetrations were made at each coordinate of a $250 \mu \mathrm{m}$ grid pattern until the distal forelimb area was circumscribed by sites evoking either proximal movements or no visible response. Small, minimal deviations were made in the $250 \mu \mathrm{m}$ grids to avoid direct damage to surface arteries or veins. When nonresponsive sites were encountered, their response to stimulation was verified later in the same mapping procedure. To verify further that the entire distal forelimb area was explored completely, a number of selected outlying sites were examined that completely flanked the distal forelimb border (see Fig. 4).

Movements were described using conventional terminology as in previous papers (Gould et al., 1986; Nudo et al., 1992). For clarity, common terminology is used where appropriate. For example, digit 1 movements are called "thumb" movements, and digits 2-5 movements are called "finger" movements.

After mapping was completed, the plastic chamber was removed, the dura was replaced with gelfilm, the bone flap was reattached with stainless steel sutures or dental acrylic, the skin was sutured, and the wound was dressed with an antibacterial agent. The animal was monitored closely until it was alert (1-2 hr), and then the animal was transferred to a temperature-controlled incubator for complete recovery.

An identical electrophysiological mapping procedure was repeated several weeks or months later after behavioral training or a control period. In our hands, repeated mapping can be performed on the same animal at least four or five times without any untoward effects such as deterioration of stimulus-evoked responses or histological signs of unintended injury. However, a thin meningeal layer often forms in the weeks or months after the initial procedure, and extra care must be taken to remove this tissue during subsequent procedures.

\section{Calculation of areal extent of movement representations}

To define unambiguous boundaries between different movement regions and thus derive reliable areal measurements, we used a computer algorithm that delineated discrete regions encompassing sites at which stimulation evoked similar movements (for further details, sec Nudo et al., 1992). Using an image analysis program (IMAGE, v1.45), the total areal extents of each movement category were derived. Finally, for each movement category, threshold current levels were tallied and analyzed.

For each animal, the areal extent of each movement category was compared for differences between pre- and post-training maps in the training group or for differences in the two sequential maps in the control group. In addition, the mean threshold current for each movement category was compared for differences in sequential maps.

\section{Histological analysis}

After completion of these experiments, boundaries of the distal foretimb representations were marked either by small, electrolytic lesions or by injection of fluorescent latex microspheres. The animal was then injected 
with a lethal dose of pentobarbital $(100 \mathrm{mg} / \mathrm{kg})$ and perfused: first with $0.9 \%$ saline, $0.2 \%$ lidocaine hydrochloride, and $1 \%$ heparin; then with $10 \%$ formalin. The brain was removed subsequently and immersed in $30 \%$ sucrose for $1-2 \mathrm{~d}$. It was cut in $50 \mu \mathrm{m}$ frontal sections. Every other section was mounted and stained with cresyl violet for cytoarchitectonic analysis. This sampling frequency was sufficient to locate each penetration site indicating the boundaries of the distal forelimb representation (electrolytic lesions or fluorescent microspheres). Tracks produced by the fine, beveled glass micropipettes used for recording stimulus-evoked movements in these studies were not detectable using these techniques.

\section{RESULTS}

\section{Behavioral results}

Three squirrel monkeys were trained to retrieve banana-flavored pellets from four food wells graded in diameter from 25 to $9.5 \mathrm{~mm}$ as outlined in Materials and Methods. On the basis of daily frame-by-frame analysis of videotapes, behavioral performance was tracked by measuring (1) the number of pellets retrieved and (2) the number of finger flexions per retrieval. Although the number of finger flexions was recorded because this movement was easy to identify on videotapes, it should be noted that each unsuccessful finger flexion consisted of a finger flexion/finger extension pair.

On the first day of training, each of the three animals readily retrieved pellets from the three largest wells. Each animal made repeated attempts (finger flexions and extensions) to retrieve pellets from the smallest well $(9.5 \mathrm{~mm}$ diameter), with varying degrees of success. In two of the three animals, performance improved rapidly on the smallest well over the course of a few days of intensive training, so that criterion performance ( 2 consecutive days with $\geq 600$ daily retrievals) was met by day 11 . Over the course of the 11 training days, the total number of daily retrievals increased, whereas the number of finger flexions per retrieval decreased (Fig. $1 A$ ). The total number of daily retrievals was significantly and negatively correlated with the number of finger flexions per retrieval $(r=-0.787, p=0.004)$.

In the third case (\#1598), criterion performance was not achieved by the end of $11 \mathrm{~d}$ of training. A mapping procedure was conducted after this animal's $11 \mathrm{~d}$ of subcriterion performance and will be presented below. After this second mapping procedure, a titration procedure was used to shape this animal's performance gradually on the smallest well. Using the titration procedurc, critcrion performance was achicved after an additional 39 $\mathrm{d}$ for a total training period of $50 \mathrm{~d}$. The number of finger flexions per retrieval for each of the three animals on days 1,11 , and 50 (case \#1598 only) is shown in Figure $1 B$.

In addition to the improvement in performance, movements became progressively more stereotypic during the course of training. By the final day of training at criterion performance levels, each of the three animals routinely retrieved pellets from the smallest well using the same movement sequence (Fig. 2). First, the pronated distal forelimb extended through the cage bars toward the target well. Second, the fingers extended and inserted into the well (typically digits 2 and/or 3; Fig. $2 A$ ). Third, the fingers flexed within the well and then stopped (Fig. $2 B$ ). Fourth, the fingers flexed a second time, and then the wrist gradually extended (Fig. $2 C, D$ ). The pellet typically was lodged between phalangeal (glabrous) surfaces of digits 2 and/or 3 and the interdigital pads (pads 2 and/or 3 ). Fifth, the wrist continued to extend as a discrete movement. Sixth, as the pellet was retrieved from the well, the forearm was gradually supinated (Fig. 2E). Finally, the distal forelimb was retracted back through the cage bars. In a later section, these movements and movement combinations used in

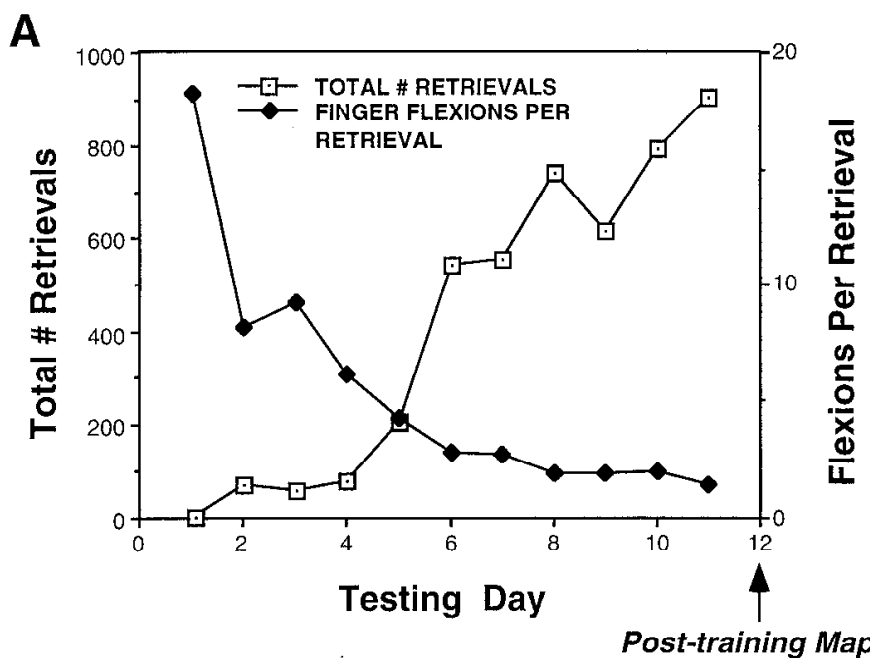

B

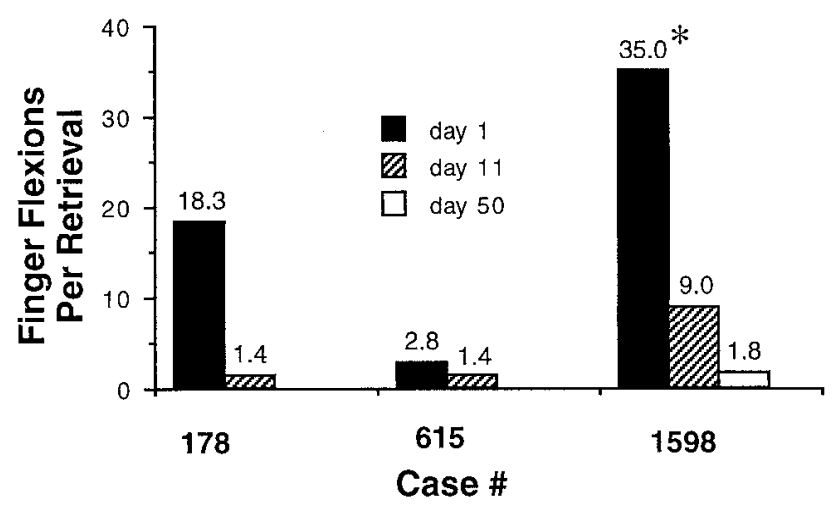

Figure 1. Performance on a Klüver board. A, Total number of successful retrievals per day (open squares) and avcrage number of finger flexions per retrieval (solid diamonds) for case \#178. This rapid acquisition was similar for case \#615. Case \#1598 required a titration procedure to ultimately achieve performance criterion. $B$, Average number of finger flexions per retrieval on the first and last days of training for each of the three animals. Asterisk indicates that data are compiled from the first two training days, because case \#1598 made no successful retrievals on the first day of training.

the task are compared with changes in movement representations after training.

It is important to note that squirrel monkeys do not exhibit functional opposition of the thumb and index finger, in contrast to many Old World primate species (Costello and Fragaszy, 1988). The pellet was withdrawn with a multidigit flexion, and prehension was achieved by the opposition of digits 2 and/or 3 with the interdigital pad. The thumb was either moved in concert with the other digits or was braced against the platform while the fingers flexed and extended. Contact between the thumb and the food pellet was rare.

\section{Organization of distal forelimb representation}

In general, the organization of the distal forelimb representation in M1 was as described in earlier reports in this species (Donoghue et al., 1992; Nudo et al., 1992). The caudal boundary was located $\sim 1-2 \mathrm{~mm}$ rostral to the central sulcus, near its lateral edge (Figs. 3, 4). The distal forelimb representation was bounded medially, rostrally, and laterally by more proximal movement representations (primarily elbow and shoulder) and caudally by a 

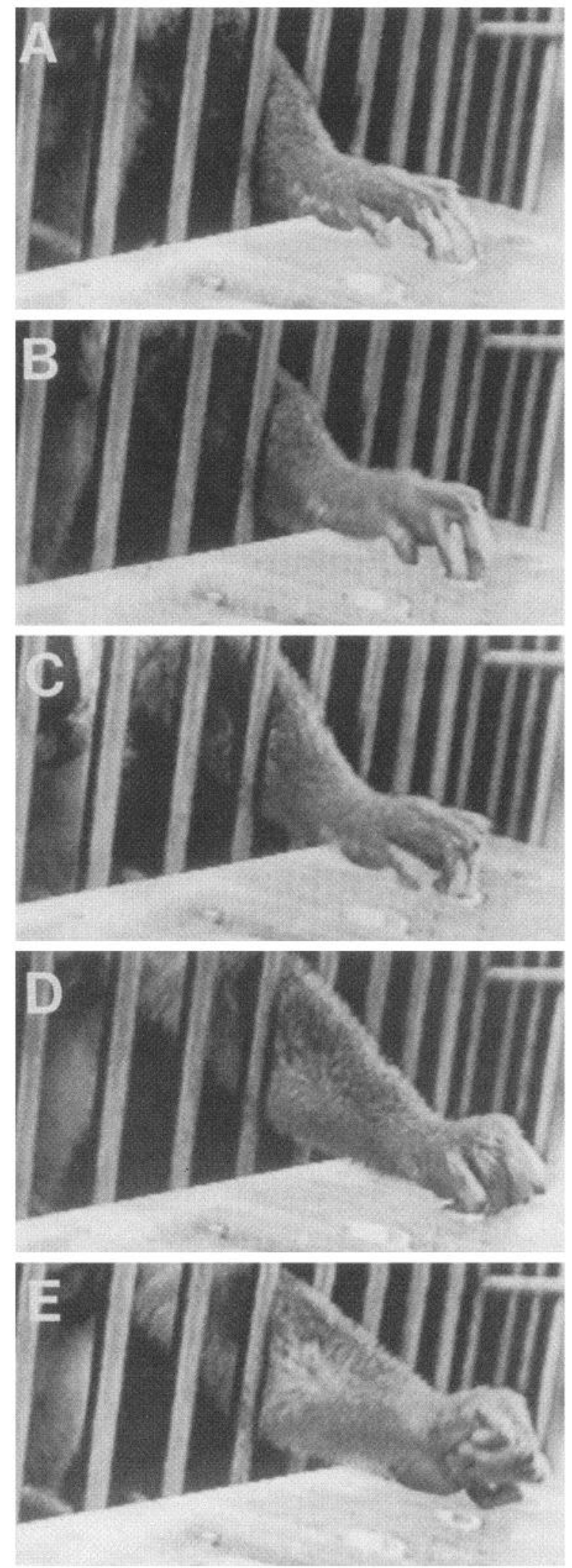

\title{
Finger Extension
}

\author{
Finger Flexion
}

\section{Finger Flexion + Wrist Extension}

\section{Wrist Extension}

\section{Forearm Supination}

Figure 2. Sequence of photographs showing movements used to retrieve a pellet from a $9.5 \mathrm{~mm}$ well (well 4 ) of a Klüver board. $A$, The fingers extended as the arm moved toward the well. $B$, The fingers flexed within the well and then stopped. $C$, A second finger flexion occurred, but before the finger flexion movement was completed the wrist extended. $D$, The wrist continued to extend as a discrete movement and then stopped when the wrist was fully extended. $E$, As the pellet was retrieved from the well, the forearm gradually supinated. 


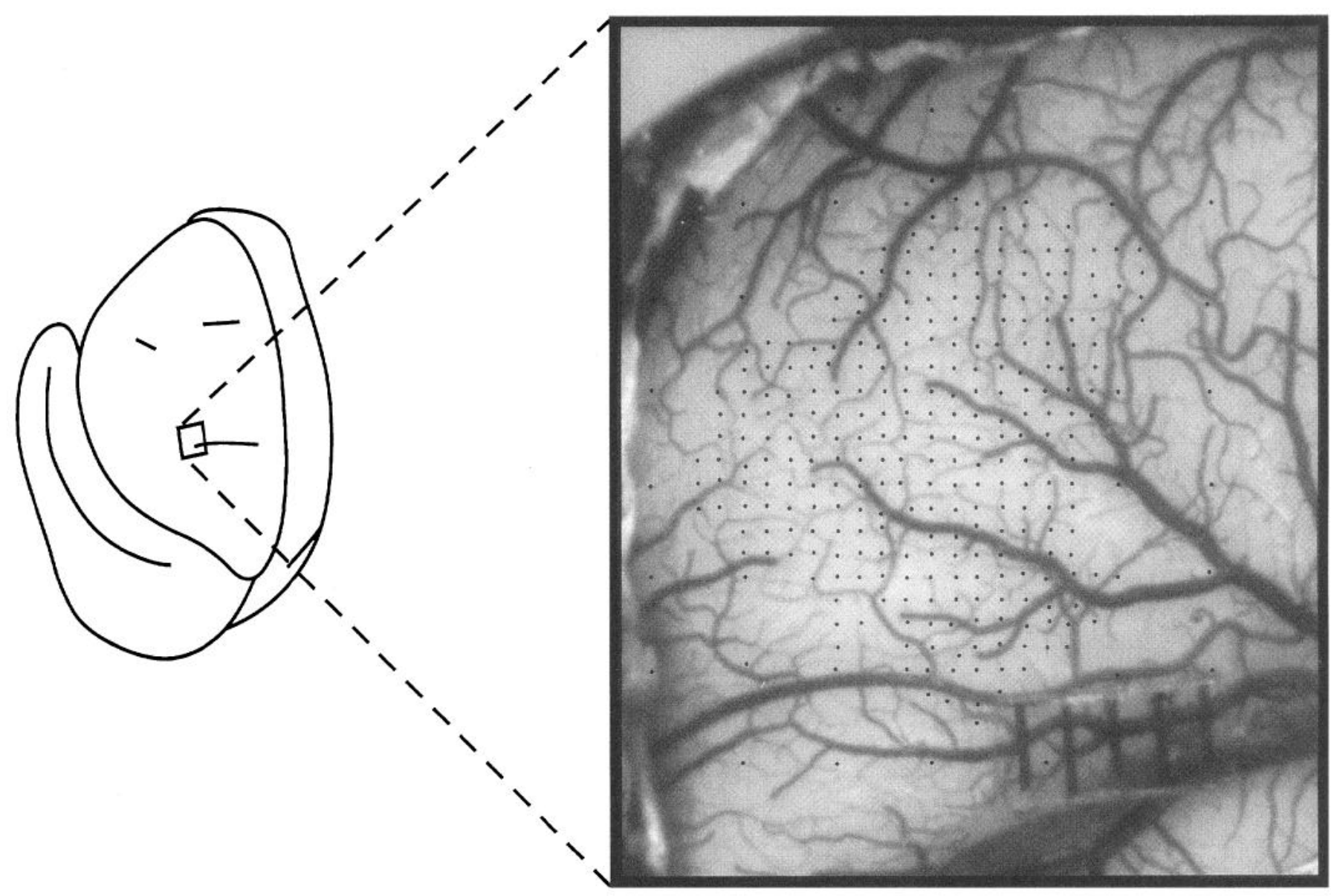

$1 \mathrm{~mm}$

Figure 3. ICMS techniques used to derive the representation of distal forelimb movements in M1 of squirrel monkey. Left, Dorsolateral view of squirrel monkey neocortex (left hemisphere), outlining the cortical territory explored in a typical motor-mapping procedure. Right, Enlarged video image of cortical surface in case \#1603 showing blood vessels used as landmarks for microelectrode insertion. Microelectrode penetration sites are indicated by small black dots.

nonresponsive zone (i.e., no movements evoked by currents $\leq 30$ $\mu \mathrm{A})$. Although the forelimb representation was usually a single contiguous sector, it could be divided occasionally into two sectors by more proximal (elbow and shoulder) representations.

Because the forelimb representation was reasonably well circumscribed on three sides by more proximal movement representations and on the remaining (caudolateral) side by an unresponsive region, the entire representation was delineated and explored in detail. Although $>99 \%$ of the sites at which stimulation evoked movements at low current levels $(\leq 30 \mu \mathrm{A})$ were found to be located within cytoarchitectonic area 4 (Nudo et al., 1992), a few responsive sites were identified subsequently on histological grounds as being within cortical area $3 \mathrm{a}$. Data from sites that were determined unambiguously to be within area 3 a (i.e., $\geq 500 \mu \mathrm{m}$ from the area $3 a / 4$ border) were eliminated from statistical analyses. The mean number of penetrations required to explore the entire forelimb representation on this $250 \mu \mathrm{m}$ sampling grain was 312.6 .

The total areal extent of distal forelimb movement maps was examined at each of four hierarchical levels of specificity, as described in a previous paper (Nudo et al., 1992). For example, distal forelimb (level 1) was divided into digit and wrist/forearm categories (level 2), digit was divided further into finger and thumb categories (level 3), and finger was divided still further into finger flexion, finger extension, finger abduction, and finger adduction categories (level 4). In addition to these single-movement categories, we also examined so-called dual-response movement categories; that is, at threshold current levels ( $\pm 2 \mu \mathrm{A})$, ICMS sometimes evoked two different movements such as finger flexion and wrist extension. Each dual-response combination was treated as a separate movement category.

Finally, we examined various combinations of these mutually exclusive categories, such as the category including all digit movements, either alone or in combination with other responses. These combined categories are referred to hereafter as inclusive categories. For example, the inclusive digit area includes the digit area, the digit + wrist/forearm area, and the digit + proximal area. For each movement category at each hierarchical level, we defined total representational area and percentage of total distal forelimb area (relative area). Thus, area measurements were performed for movement categories at hierarchy level 1 (i.e., distal forelimb), level 2 (i.e., digit, wrist/forearm), level 3 (i.e., finger, thumb, etc.), and level 4 (i.e., finger flexion, finger extension, finger abduction, etc.).

The distal forelimb area was highly variable in extent among individual animals. The mean areal extent for the 12 maps in six 


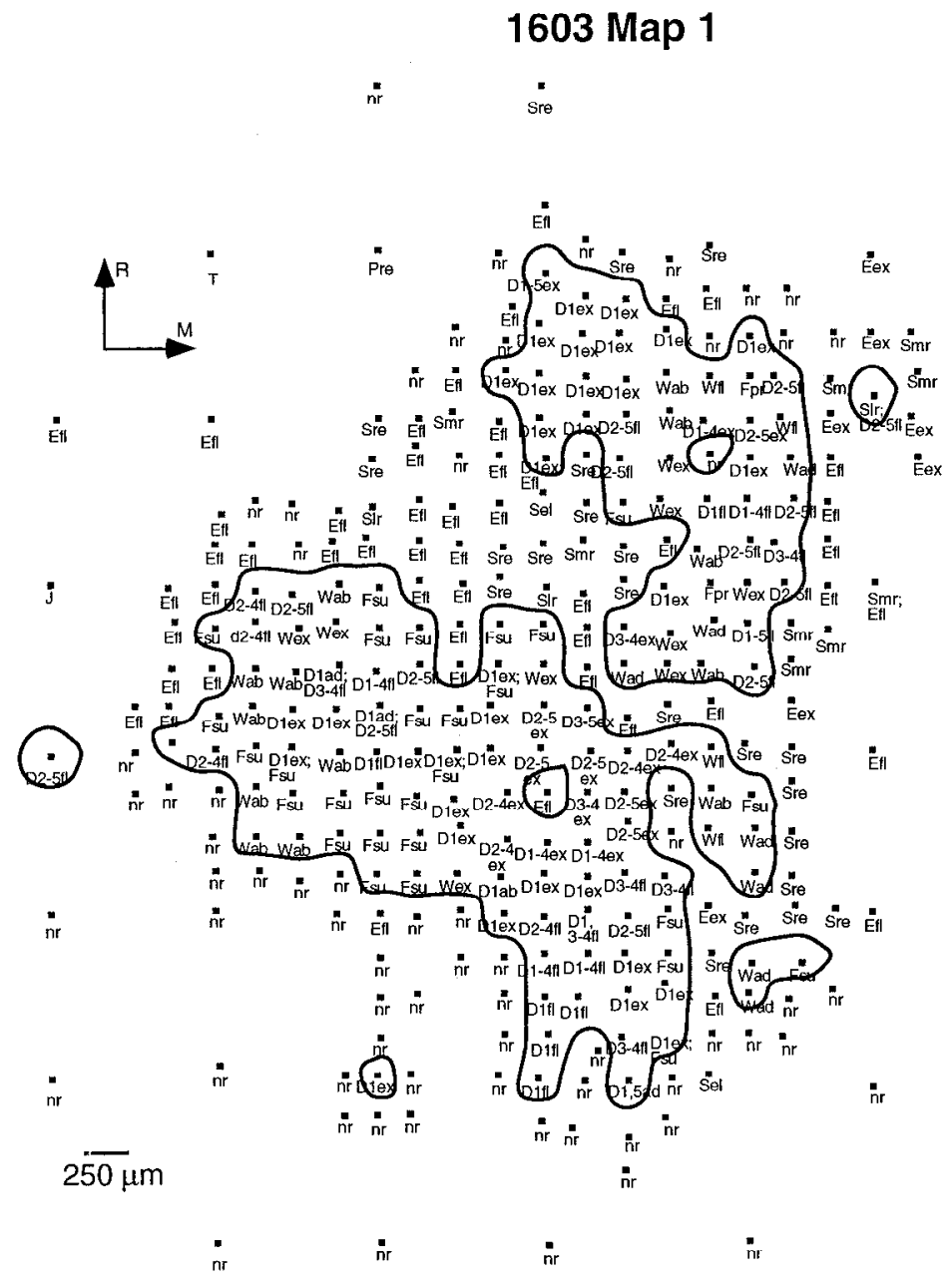

individual animals was $12.08 \mathrm{~mm}^{2}$ and ranged from 7.47 to 14.46 $\mathrm{mm}^{2}$ (Tablc 1). As reportcd in a prcvious paper (Nudo ct al., 1992), no significant correlation was found between distal forelimb representational area and body weight $(r=0.144, p=0.79$ for animals reported in the present study).

The representational area for movement classes in hierarchical levels 2-4 was also highly variable. For example, the digit area averaged $6.37 \mathrm{~mm}^{2}$ among the 12 representational maps, ranging from 2.74 to $9.28 \mathrm{~mm}^{2}$. On average, the digit area accounted for $52.2 \%$ of the total distal forelimb representation. The wrist/forearm area averaged $4.66 \mathrm{~mm}^{2}$ and ranged from 2.46 to $6.71 \mathrm{~mm}^{2}$. On average, the wrist/forearm area accounted for $38.6 \%$ of the total distal forelimb representation. The digit + wrist/forearm dual-response area averaged $0.65 \mathrm{~mm}^{2}$ and ranged from 0.05 to $1.56 \mathrm{~mm}^{2}$. On average, the digit + wrist/forearm area accounted for $5.6 \%$ of the total distal forelimb area (Table 1).

\section{Relative stability of movement representations in control cases}

Movement representations were not identical between mapping procedures in control cases (Fig. 5). Considerable site-by-site variation was evident throughout the distal representation. However, in general, the topography of distal forelimb representation remained relatively constant. With respect to the surface vasculature, the distal forelimb representation was confined to the same cortical territory in both maps. Also, the general locations of mosaical clusters of movement categories were similar in the two maps. Still further, the total distal forelimb representation was relativcly constant. Finally, very little change was cvident in the percentage of total area occupied by each movement category. In the present sample, the variability between animals appeared to be much greater than the variability between mapping procedures of the same animal (see Fig. 5). No systematic areal changes were apparent.

\section{Changes in movement representations after digit training}

Initial examination of distal forelimb representations derived in pre- and post-training maps revealed several apparent changes in movement topography. For example, expressed as a percentage of total distal forelimb area, the digit area increased in two of three cases, whereas the wrist/forearm area decreased in each of the cases (Fig. 6). Thus, despite the mosaical representation of distal forelimb movements and the large variability across individuals in representational area, it appears that systematic changes in individual movement representations occur in $\mathrm{M} 1$ after motor training.

To determine whether apparent changes between pre- and post-training representational maps were significantly different from control procedures, each of the mutually exclusive movement categories listed in Table 1, as well as various combinations of these movement categories, was submitted to statistical analyses. Movement categories for which total areal representation was $<2 \%$ of the total distal forelimb area in the 12 maps were 
Table 1. Mean area and percent of total distal forelimb area for various movement classes

\begin{tabular}{|c|c|c|c|c|}
\hline Movement & $\operatorname{Arca}\left(\mathrm{mm}^{2}\right)$ & $\mathrm{SD}$ & $\%$ Total & $\mathrm{SD}$ \\
\hline Distal forelimb & 12.08 & 2.43 & 100.00 & - \\
\hline Digit & 6.37 & 1.95 & 52.18 & 11.50 \\
\hline Wrist/forearm & 4.66 & 1.45 & 38.60 & 8.89 \\
\hline Digit + wrist/forearm & 0.65 & 0.48 & 5.60 & 4.32 \\
\hline Digit + proximal & 0.23 & 0.28 & 2.09 & 2.74 \\
\hline Wrist/forearm + prox. & 0.18 & 0.23 & 1.53 & 1.72 \\
\hline Fingers & 4.48 & 1.17 & 36.99 & 5.76 \\
\hline Thumb & 1.89 & 1.11 & 15.15 & 8.82 \\
\hline Wrist & 3.36 & 1.21 & 27.77 & 7.46 \\
\hline Forearm & 1.30 & 0.49 & 10.84 & 3.93 \\
\hline Finger + wrist & 0.37 & 0.36 & 3.24 & 3.16 \\
\hline Finger + forearm & 0.01 & 0.03 & 0.13 & 0.36 \\
\hline Finger + proximal & 0.14 & 0.20 & 1.40 & 2.43 \\
\hline Thumb + wrist & 0.08 & 0.09 & 0.70 & 0.85 \\
\hline Thumb + forearm & 0.20 & 0.20 & 1.64 & 1.49 \\
\hline Thumb + proximal & 0.09 & 0.13 & 0.70 & 1.02 \\
\hline Wrist + proximal & 0.11 & 0.16 & 0.95 & 1.17 \\
\hline Forearm + proximal & 0.09 & 0.19 & 0.72 & 1.37 \\
\hline Finger flexion & 2.06 & 0.50 & 18.28 & 7.79 \\
\hline Finger extension & 2.08 & 1.51 & 16.23 & 11.40 \\
\hline Finger abd./add. & 0.34 & 0.53 & 2.49 & 3.74 \\
\hline Thumb flexion & 0.44 & 0.43 & 3.55 & 3.22 \\
\hline Thumb extension & 1.23 & 0.65 & 9.91 & 5.52 \\
\hline Thumb abd./add. & 0.21 & 0.24 & 1.69 & 1.91 \\
\hline Wrist flexion & 0.17 & 0.23 & 7.01 & 4.57 \\
\hline Wrist extension & 1.78 & 0.99 & 15.25 & 8.65 \\
\hline Wrist adduction & 0.98 & 0.87 & 7.58 & 5.80 \\
\hline Wrist abduction & 0.44 & 0.35 & 3.60 & 3.06 \\
\hline Forearm pronation & 0.38 & 0.27 & 3.12 & 2.29 \\
\hline Forearm supination & 0.91 & 0.40 & 7.67 & 3.57 \\
\hline Fing. flexion + w.ext. & 0.24 & 0.25 & 2.24 & 2.65 \\
\hline Thumb fl. I w.cxt. & 0.03 & 0.06 & 0.27 & 0.45 \\
\hline Thumb ext. + fa.sup. & 0.14 & 0.17 & 1.20 & 1.38 \\
\hline Other finger (dual) & 0.22 & 0.23 & 1.71 & 2.37 \\
\hline Other thumb (dual) & 0.26 & 0.52 & 2.16 & 4.56 \\
\hline Other wrist (dual) & 0.33 & 0.64 & 2.61 & 4.42 \\
\hline Other forearm (dual) & 0.23 & 0.23 & 1.96 & 1.83 \\
\hline
\end{tabular}

Abbreviations: prox., Proximal; abd., abduction; add., adduction; w.ext., wrist extension; fl., flexion; ext., extension; fa.sup., forearm supination.

eliminated from further analysis. Thus, the total number of movement categories submitted for statistical analysis was 57. Repeated-measures ANOVA was used to test differences between representational areas in the two maps as a function of treatment condition (training vs control conditions). When significant map $x$ training condition interactions were found, paired comparisons (two-tailed $t$ tests, $p=0.05$ ) were used as post hoc tests to examine further the differences between pre- and posttraining representational areas. To normalize percentage entries, arcsin transformations were used on these values before statistical testing (Zar, 1984).

When the entire distal forelimb representation was examined, representational area increased in each of the three training cases by an average of $0.48 \mathrm{~mm}^{2}$, corresponding to an average percent- age increase of $5.0 \%(3.9,9.5$, and $1.6 \%$ in cases 178,615 , and 1598 , respectively). This increase was not significantly different from control $(p<0.05)$. However, when distal forelimb movements were subdivided (hierarchical levels 2-4 and their combinations), several significant differences were found between training and control procedures. Because the total distal forelimb area did not change significantly, statistical analyses are reported for relative representational areas expressed as a percentage of total distal forelimb area. Of the 57 movement categories examined, seven significant differences were found between pre- and posttraining representational areas. Table 2 lists these statistically significant results as well as the results of three additional statistical tests that approached significance.

The areal extent of four single-movement representations differed significantly hetween training and control procedures. As shown in Table 2, the (inclusive) digit extension representation increased in relative area in training animals compared with controls. In contrast, the wrist, wrist abduction, and (inclusive) wrist abduction representations decreased in relative area in training animals compared with controls. In addition, increases in the (inclusive) finger representation and decreases in the (inclusive) wrist representation approached statistical significance.

The relative areal extent of two dual-response representations differed significantly between training and control procedures. The digit + wrist/forearm and finger flexion + wrist extension representations increased in areal extent in training animals. After training, the finger flexion + wrist extension representation increased nearly 2.5 -fold from 0.12 to $0.41 \mathrm{~mm}^{2}$, or from 1.6 to $4.2 \%$ of the total distal forelimb area. In addition, incrcascs in the digit flexion + wrist extension representation approached statistical significance.

Neither the finger flexion nor the finger extension representations were significantly different between training and control procedures. However, when these representations were combined, the resulting finger flexion/extension representation increased in relative areal extent in training animals compared with controls. In training animals, the finger flexion/extension representation increased from 3.72 to $5.35 \mathrm{~mm}^{2}$, a $44 \%$ increase, whereas in control animals this representation decreased from 4.28 to $3.97 \mathrm{~mm}^{2}$, a $7 \%$ decrease. Expressed as a percentage of distal forelimb area, the finger flexion/extension representation increased significantly from 33.1 to $46.2 \%$ in training animals, whereas the representation remained relatively constant in controls $(33.85-33.82 \%)$.

In summary, digit training resulted in statistically significant changes in the relative areal extent of several movement representations, including increases in digit extension, finger flexion + wrist extension, and finger flexion/extension representations and decreases in wrist abduction representations. Several of these significant changes are illustrated graphically in Figure 8 . It should be noted that the absolute (as opposed to relative) areal extents of many of these same movement representalions clanged significantly but are not reported here.

\section{Changes in movement thresholds after digit training}

To determine whether any of the changes in representational area were related to changes in the thresholds for evoking movements, thresholds for each of the 57 movement categories were determined for each of the three training cases. Two movement categories showed significant increases in movement threshold. Thresholds for finger movements increased in each of the three training cases from an average of 12.94 to $15.46 \mu \mathrm{A}(t=6.46$, 


\section{CONTROL CASES: DIGIT, WRIST/FOREARM REPRESENTATIONS}
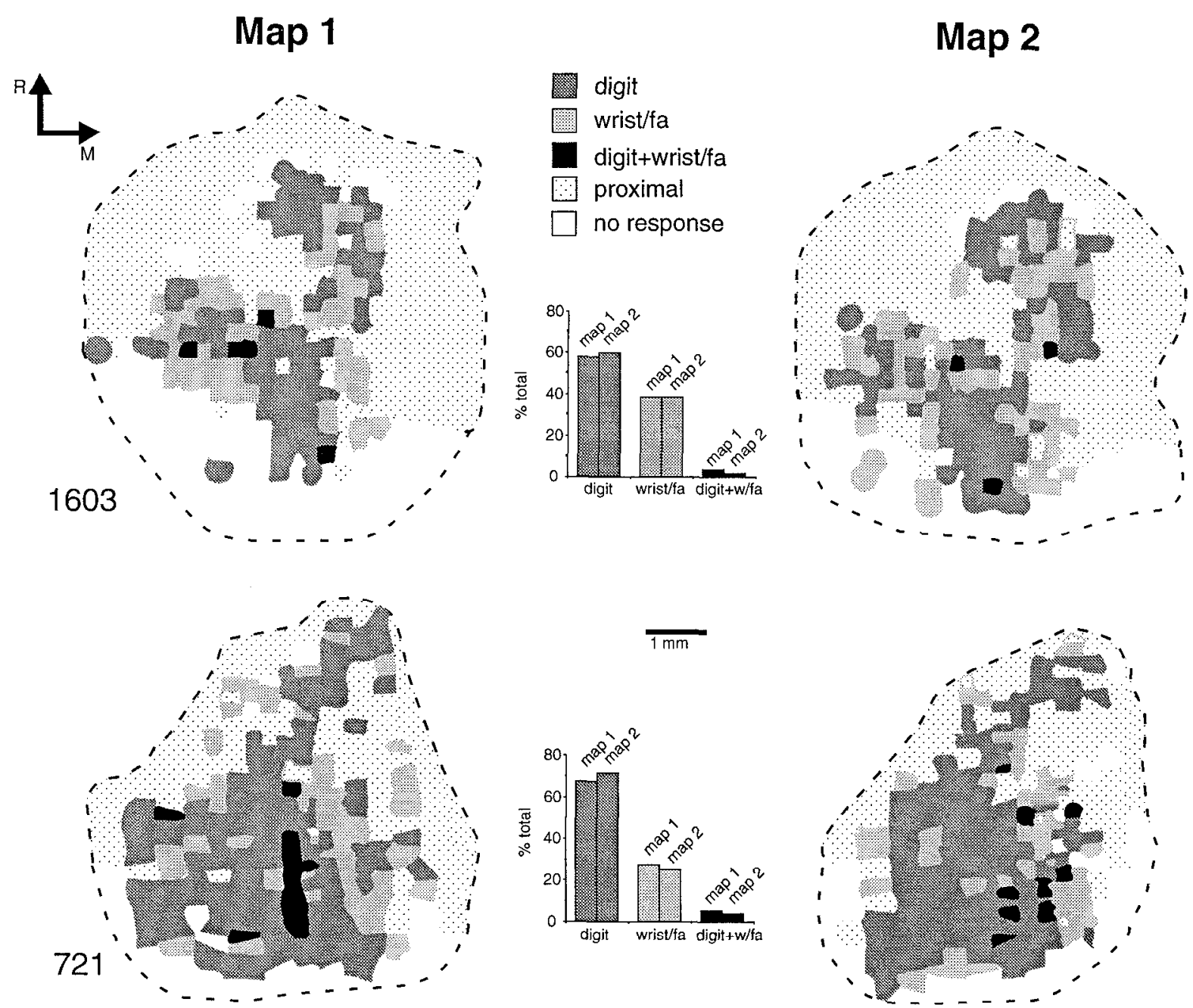

155
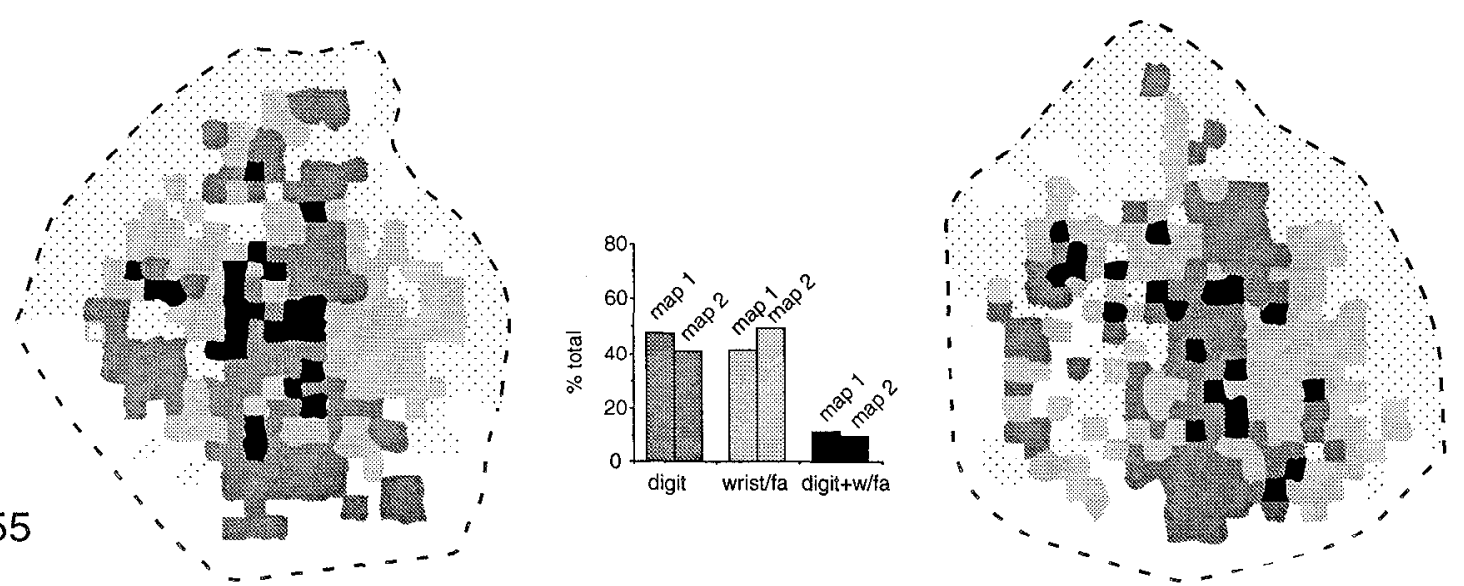

Figure 5. Representation of the distal forelimb in cortical area 4 derived from two separate mapping procedures in three control animals. In this illustration, distal forelimb movements have been broadly categorized as digit (dark gray), wrist/forearm (wrist/fa, light gray), digit + wrist/forearm (digit $+w / f a$, black), or proximal (stippled) movements. Nonresponsive areas are shown in white. For simplification, digit + proximal movements are included with digit; wrist/forearm + proximal movements are included with wrist/forearm. Dashed lines indicate extent of explored region. All maps are depicted in the left hemisphere. 

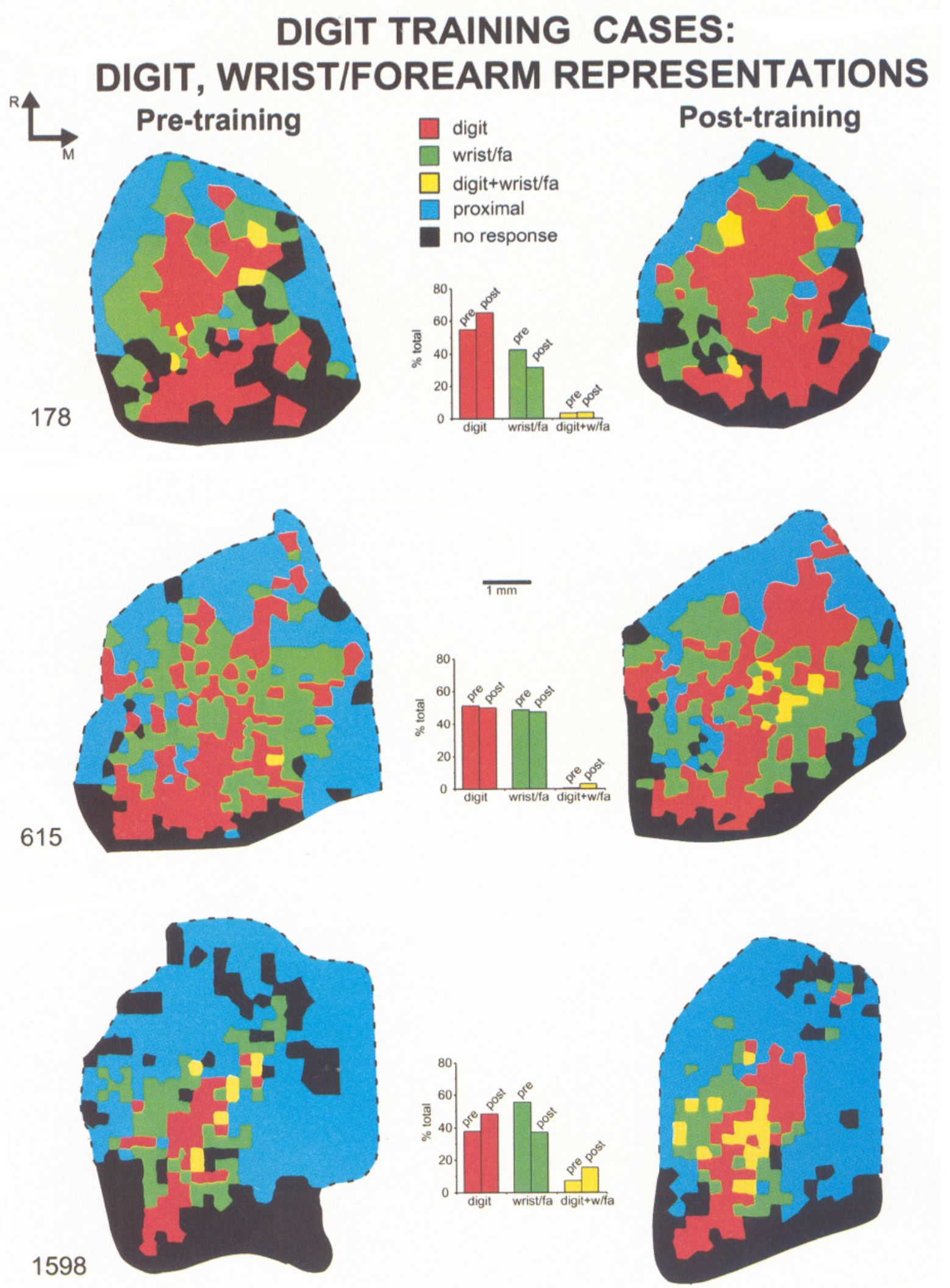

Figure 6. Representation of the distal forelimb in cortical area 4 derived from pre- and post-training mapping procedures in three training animals. In this illustration, distal forelimb movements have been broadly categorized as digit (red), wrist/forearm (green), digit + wrist/forearm (yellow), or proximal (blue) movements. Nonresponsive areas are shown in black. Abbreviations as in Figure 5. 


\section{DIGIT TRAINING CASES: FINGER EXTENSION, FINGER FLEXION, WRIST ABDUCTION REPRESENTATIONS}

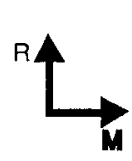

178

\section{Pre-training}

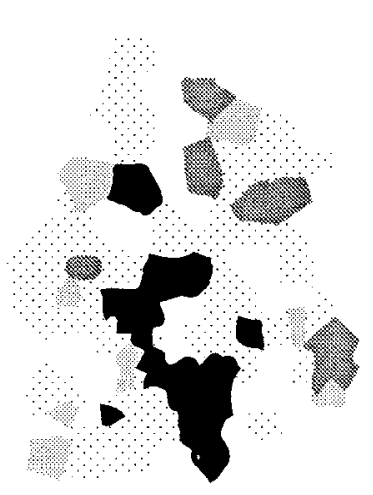

615
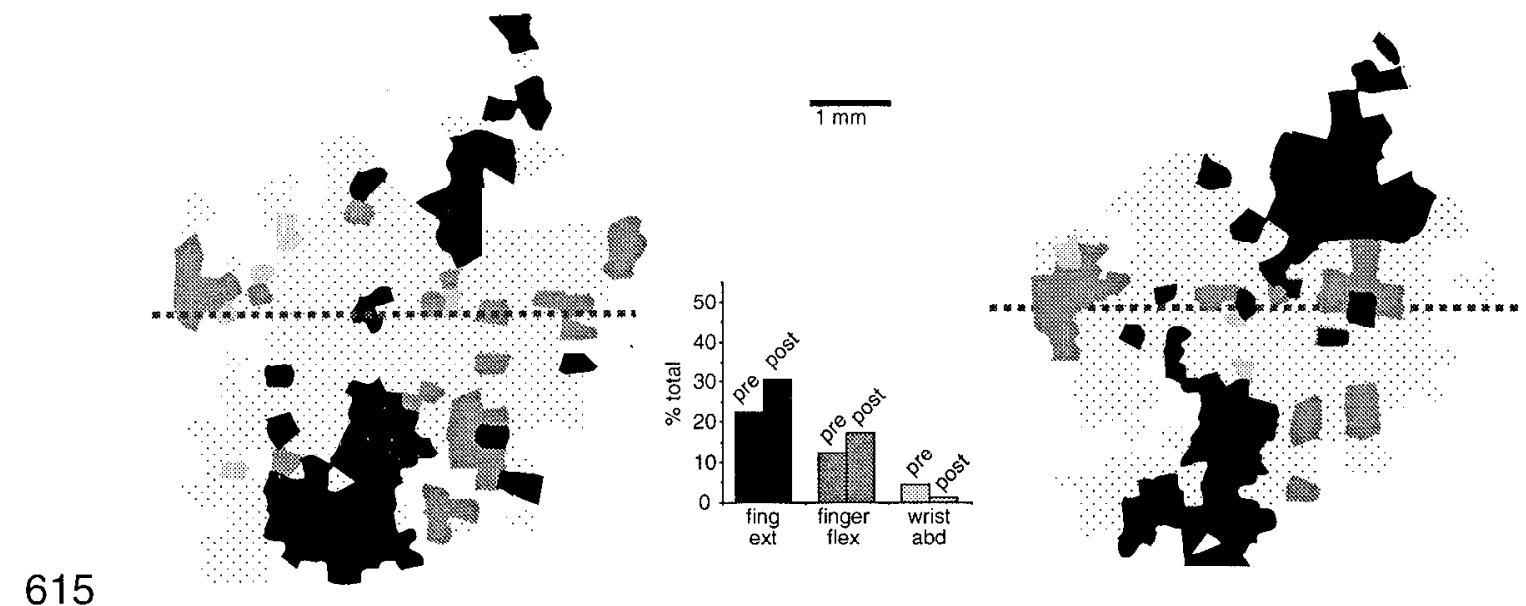

Post-training
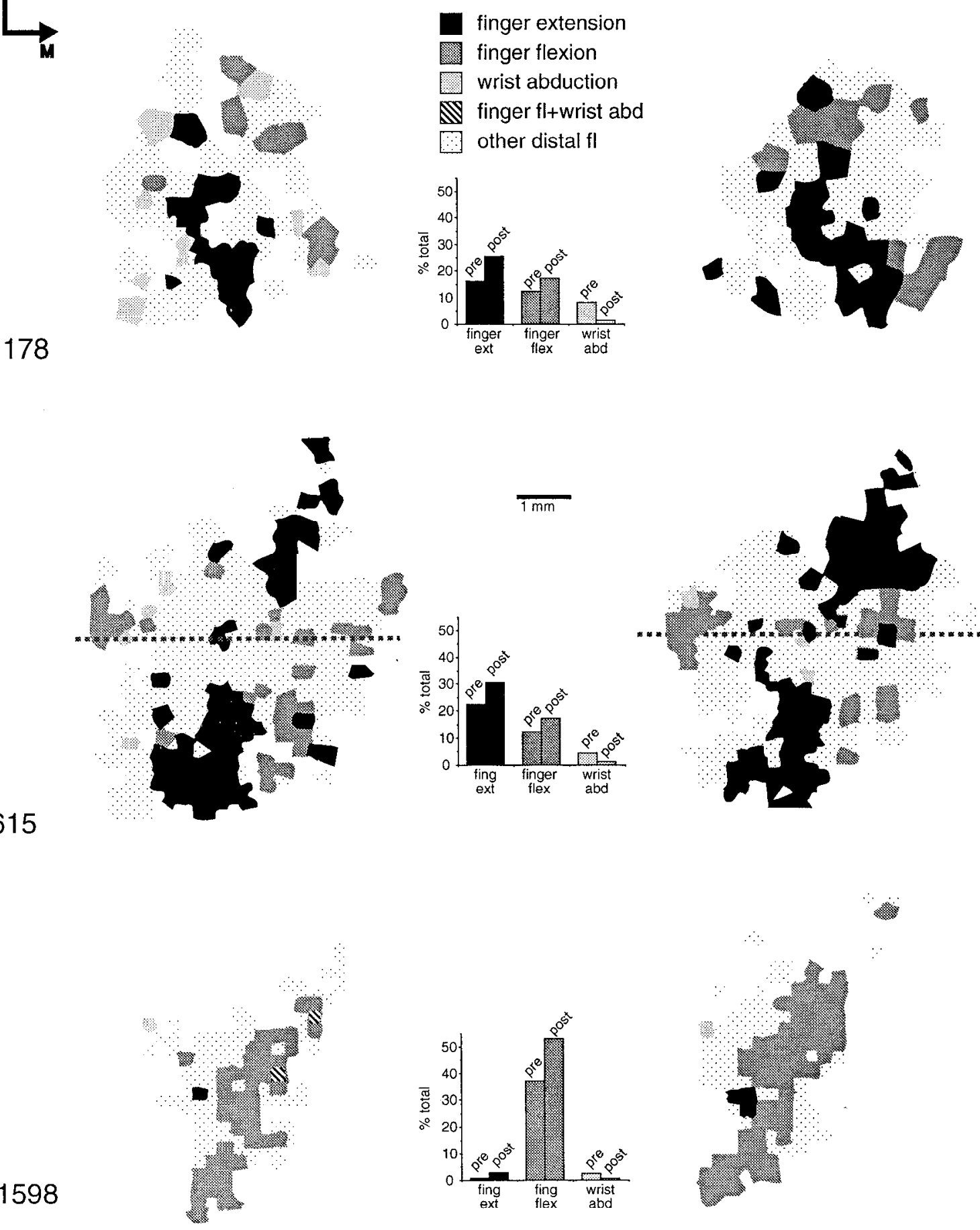

Figure 7. Representation of finger extension, finger flexion, and wrist abduction movements in cortical area 4 derived from pre- and post-training mapping procedures. In this illustration, finger extension movements are shown in black, thumb flexion movements are shown in dark gray, wrist abduction movements are shown in light gray, and finger flexion + wrist abduction movements are indicated by diagonal lines. For simplification, other dual-responses are included in single-movement categories. In bar graphs, finger flexion + wrist abduction dual-responses are included in each of the individual movement categories. Despite a large variation in movement representations among individuals, similar changes were evident in each of the three cases. That is, the relative area devoted to both finger extension and finger flexion movements increased in each of the three cases; the relative area devoted to wrist abduction movements decreased in each of the three cases. Dotted line in case \#615 denotes division of distal forelimb representation into caudal and rostral sectors. Dashed lines indicating extent of explored region (Figs. 5,6 ) have been eliminated in this and subsequent figures for clarity. $f$, Flexion; ext, extension; flex, flexion; abd, abduction. 
$\%$ Distal forelimb area means $\pm \mathrm{SD}$

\begin{tabular}{|c|c|c|c|c|c|c|}
\hline \multirow[b]{3}{*}{ Movement } & \multirow{2}{*}{\multicolumn{2}{|c|}{ ANOVA results ${ }^{a}$}} & \multirow{2}{*}{\multicolumn{2}{|c|}{ Digit training group }} & \multirow{2}{*}{\multicolumn{2}{|c|}{ Control group }} \\
\hline & & & & & & \\
\hline & $F$ & $P$ & Map 1 & Map 2 & Map 1 & Map 2 \\
\hline Digit + wrist/forearm & $9.95^{*}$ & 0.034 & $3.58 \pm 3.38$ & $7.23 \pm 6.77$ & $6.61 \pm 4.07$ & $5.00 \pm 4.10$ \\
\hline Wrist & $10.14^{*}$ & 0.033 & $34.56 \pm 6.22$ & $26.68 \pm 8.68$ & $21.92 \pm 2.68$ & $27.91 \pm 7.89$ \\
\hline Finger (inclusive) & 6.93 & 0.058 & $34.92 \pm 5.27$ & $47.88 \pm 7.30$ & $42.97 \pm 8.47$ & $41.31 \pm 7.14$ \\
\hline Wrist (inclusive) & 6.17 & 0.068 & $37.63 \pm 8.91$ & $32.94 \pm 9.15$ & $26.66 \pm 6.15$ & $33.29 \pm 9.61$ \\
\hline Finger flexion + wrist extension & $14.39^{*}$ & 0.019 & $1.55 \pm 2.38$ & $4.21 \pm 4.10$ & $2.49 \pm 2.37$ & $0.71 \pm 0.61$ \\
\hline Finger flexion/extension & $8.25^{*}$ & 0.045 & $33.13 \pm 4.79$ & $46.23 \pm 8.56$ & $33.85 \pm 8.93$ & $33.82 \pm 13.91$ \\
\hline Digit extension (inclusive) & $7.93^{*}$ & 0.048 & $22.81 \pm 16.86$ & $27.04 \pm 17.49$ & $37.23 \pm 7.01$ & $35.96 \pm 6.60$ \\
\hline Digit flexion + wrist extension & 7.28 & 0.054 & $1.55 \pm 2.38$ & $4.94 \pm 4.20$ & $2.49 \pm 2.37$ & $1.06 \pm 0.43$ \\
\hline Wrist abduction & $19.25^{*}$ & 0.012 & $4.51 \pm 3.74$ & $0.84 \pm 0.73$ & $4.43 \pm 3.62$ & $4.62 \pm 2.94$ \\
\hline Wrist abduction (inclusive) & $13.52^{*}$ & 0.021 & $5.04 \pm 2.98$ & $1.09 \pm 0.31$ & $4.55 \pm 3.55$ & $4.62 \pm 2.94$ \\
\hline
\end{tabular}

${ }^{a}$ ANOVA (with repeated measures) was used to test differences between relative representational area in the two maps as a function of treatment condition (map X training condition interactions). Statistically significant $F$-values indicate that areal changes between Map 1 and Map 2 differ between training and control groups. *Significant
differences at $p<0.05$.

$p=0.023$ ). Likcwisc, thresholds for finger extension movements increased in each of the three training cases from an average of 10.41 to $14.20 \mu \mathrm{A}(t=4.45, p=0.047)$; that is, more current was required to evoke finger and finger extension movements after training. No significant differences in threshold were found for any of the movement categories in control cases.

It is possible that some of the changes in representational area observed in these procedures were attributable to systematic changes in movement thresholds. For example, if more current was required to evoke finger extension movements after training, then an expanded finger extension representation may reflect the "unmasking" of a larger finger representation zone that was not seen because of our definition of movements at just-threshold current levels. To examine this possibility in more detail, in one case (\#1598) at each site we recorded movements evoked at a fixed $20 \mu \mathrm{A}$ current level in addition to the threshold current. At threshold current levels, the finger extension arca incrcascd from $0.06 \mathrm{~mm}^{2}$ before training to $0.24 \mathrm{~mm}^{2}$ after training, a fourfold increase. At the fixed suprathreshold current level of $20 \mu \mathrm{A}$, the finger extension area increased from 0.19 to $0.57 \mathrm{~mm}^{2}$, a threefold increase. Thus, even at fixed suprathreshold current intensities, the areal extent of finger extension representations increased.

The possibility that systematic changes in movement thresholds play a role in expanded representations of dual-response areas was also explored by examining fixed suprathreshold current responses. As shown in Figure 9, increasing current from threshold levels to $20 \mu \mathrm{A}$ resulted in a small increase in the areal representations of finger flexion and wrist extension. However, after training the areal extent of the finger flexion zone, but not the wrist extension zone, increased substantially. This was evident at both threshold and $20 \mu \mathrm{A}$ current levels. Perhaps surprisingly, the average current required to evoke finger flexion increased slightly, whereas the average current required to evoke wrist extension and finger flexion + wrist extension decreased substantially.

\section{Rostral-caudal differences in use-dependent reorganization}

Although changes in the topography of movement representations occurred throughout the distal forelimb field, it appeared that some changes were greater in the rostral sector of area 4 . For
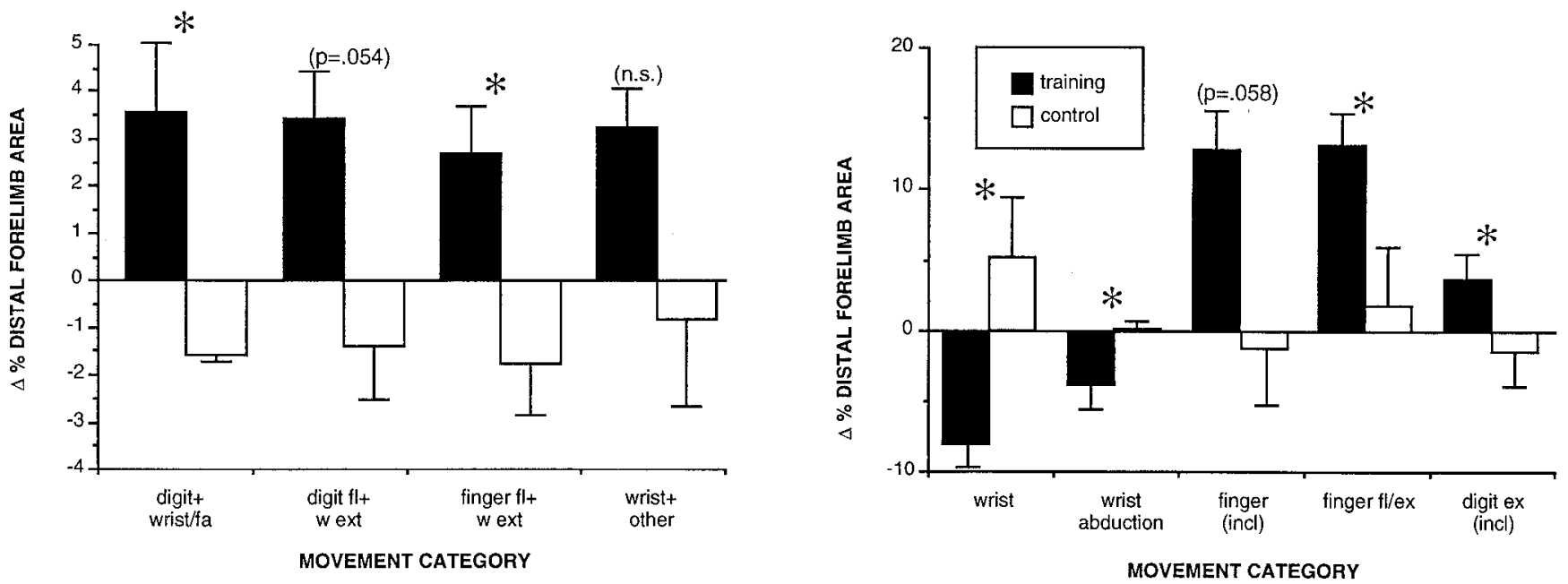

Figure 8. Changes in percentage of distal forelimb area devoted to various movement representations in M1 aftcr digit-training and control procedures. Asterisks indicate statistically significant differences between training and control procedures using repeated-measures ANOVA $(p<0.05)$. $f a$, Forearm; $w$ ext, wrist extension; $n . s$., not significant; incl, inclusive; $f$, flexion; ex, extension. 
A

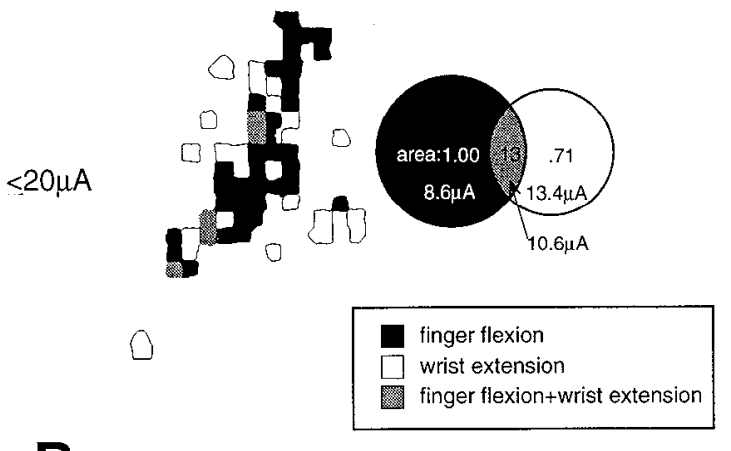

B

$20 \mu \mathrm{A}$

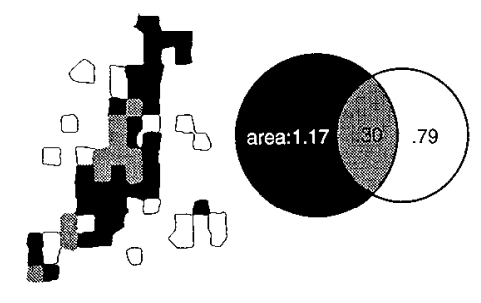

a
POST-TRAINING
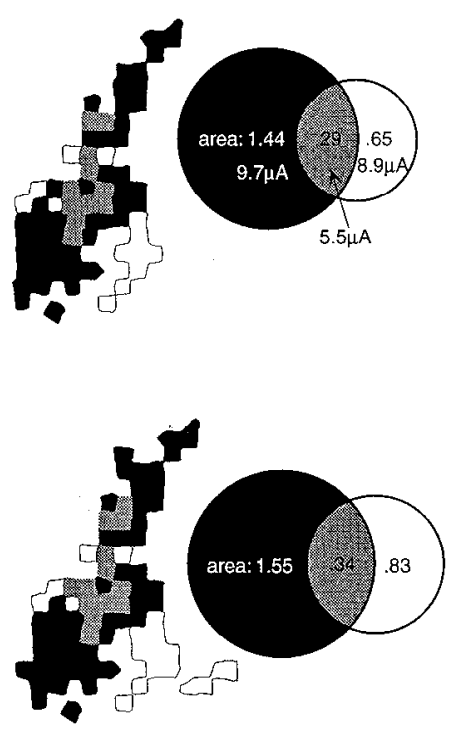

Figure 9. Representation of finger flexion and wrist extension in case \#1598 at $(A)$ threshold current levels and $(B)$ a fixed current level of 20 $\mu$ A. In $A$, only sites at which threshold was $\leq 20 \mu \mathrm{A}$ were considered. Venn diagrams depict normalized areas and mean thresholds for each movement category. Finger flexion and wrist extension areas are inclusive. Increasing current from threshold levels to $20 \mu \mathrm{A}$ resulted in a small increase in the areal representations of finger flexion and wrist extension. After training, the finger flexion zone as well as the finger flexion + wrist extension zone increased substantially in total areal extent. Training also resulted in a decrease in the average threshold for evoking wrist extension in this case (\#1598). These results suggest that, after training, increased representational area for finger flexion + wrist extension occurs by (1) expansion of finger flexion representation into wrist extension and other territories and (2) decreased thresholds for wrist extension. example, in case \#615 a large, contiguous digit represen tation appeared in the rostral portion of the distal forelimb field after motor training (Figs. 6, 7). This enlarged representation appeared to invade the adjacent wrist/forearm and elbow/shoulder representations. To determine whether these apparent differences were statistically significant, the distal forelimb representation was subdivided into rostral and caudal halves. First, in each animal the rostral and caudal limit of the distal forelimb representation derived in the pretraining map was demarcated. A line was then drawn bisecting the distal forelimb representation into rostral and caudal halves. This line was then drawn over the post-training map at the same anatomical location with respect to the surface vasculature. Paired $t$ tests were used as post hoc analyses to examine rostral-caudal differences.

Of the movement categories that were significantly or nearly significantly different between pre- and post-training maps (Table 2 ), two were significantly different in the rostral, but not the caudal, sector (Fig. 10). First, the relative finger flexion dualresponse area (finger flexion + other) was larger in each of the three post-training maps in both rostral and caudal halves. However, this increase was significant only in the rostral half. Second, the relative wrist abduction area decreased in each of the three post-training maps in both the rostral and caudal halves. This decrease was significant only in the rostral half. In addition, the relative finger (inclusive) area increased in both the rostral and caudal halves. This decrease was significant only in the rostral half.

\section{Progressive changes in motor representations during initial acquisition, extinction, and reacquisition of a skilled motor task}

To determine the progression of changes in motor representations during the acquisition of a behavioral task and whether these changes are reversible, one animal underwent five complete mapping procedures spanning $>500 \mathrm{~d}$ : (1) baseline map, (2) posttraining phase I map (subcriterion performance), (3) post-training phase II map (criterion performance), (4) postextinction map, and (5) post-reacquisition map.

\section{Behavioral performance}

Phase I training. Phase I training was conducted using the Klüver board for $11 \mathrm{~d}$. This training procedure was identical to that described for cases \#615 and \#178. However, the behavioral performance of case \#1598 at the end of the training phase was markedly lower. On day 11 , cases \#615 and \#178 retrieved $>600$ pellets (criterion performance), whereas case \#1598 retrieved only 85 pellets. Also, on day 11 cases \#178 and \#615 each made an average of 1.4 flexions per retrieval on the smallest well (well 4), whereas case \#1598 made an average of 9.0 flexions per retrieval (Fig. 1). The next day, a second motor-mapping procedure was conducted.

Phase II training. Phase II training included a titration procedure to encourage gradually more efficient retrieval of pellets from progressively smaller wells in the Klüver board. After $39 \mathrm{~d}$ of phase II training, criterion performance was reached. On the last day of phase II training, both the number of retrievals (772) and the finger flcxions pcr retricval (1.8) were similar to the perfor mance levels of cases \#178 and \#615 after $11 \mathrm{~d}$ of phase I training. On the day after the phase II training, a third motormapping procedure was conducted.

Extinction. The animal was placed in its home cage for 4 months. During this time, no specific behavioral training was conducted. After this extinction period, a fourth motor-mapping procedure was conducted. After an ad libitum feeding period of 27 $\mathrm{d}$, and then $2 \mathrm{~d}$ of food restriction, a behavioral probe was conducted on well 4 to gauge the effects of the extinction period on the animal's motor performance. The results of this behavioral probe showed that the animal's performance had reverted back to near-pretraining levels; that is, the number of retrievals fell to 122 pellets, and the flexions per retrieval rose to 4.75 .

Reacquisition. Four and one-half months after the extinction map was generated, reacquisition training began. The procedure 
A

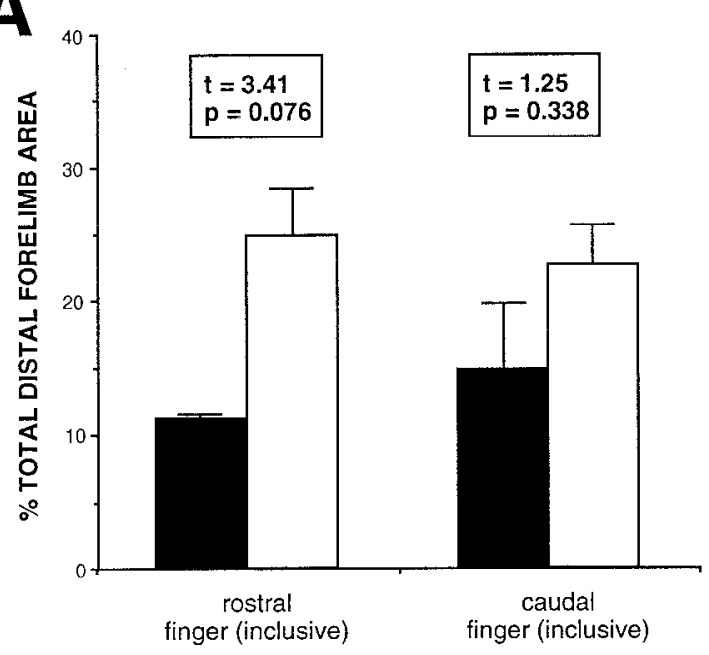

B

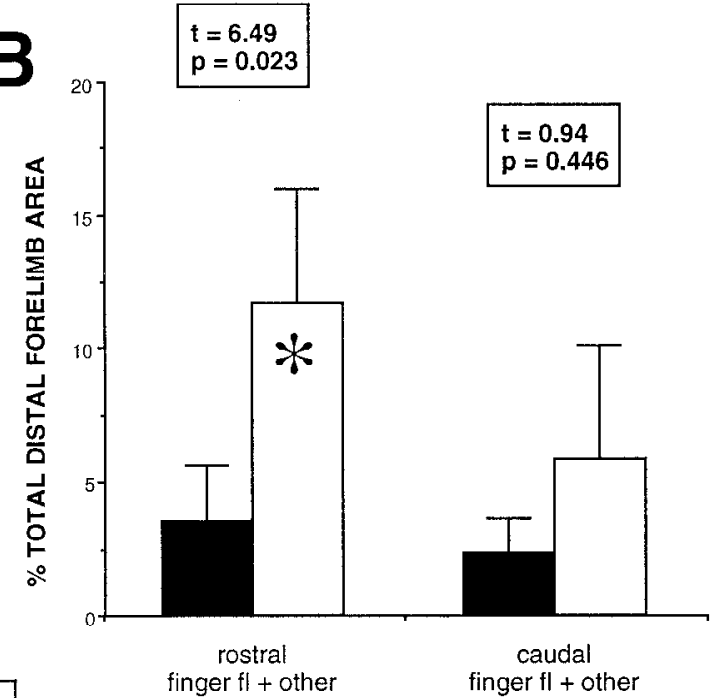

C

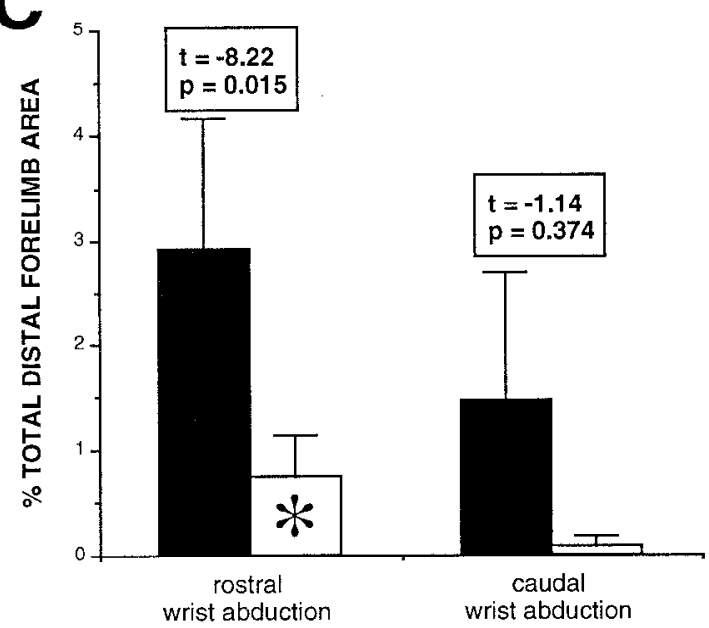

D

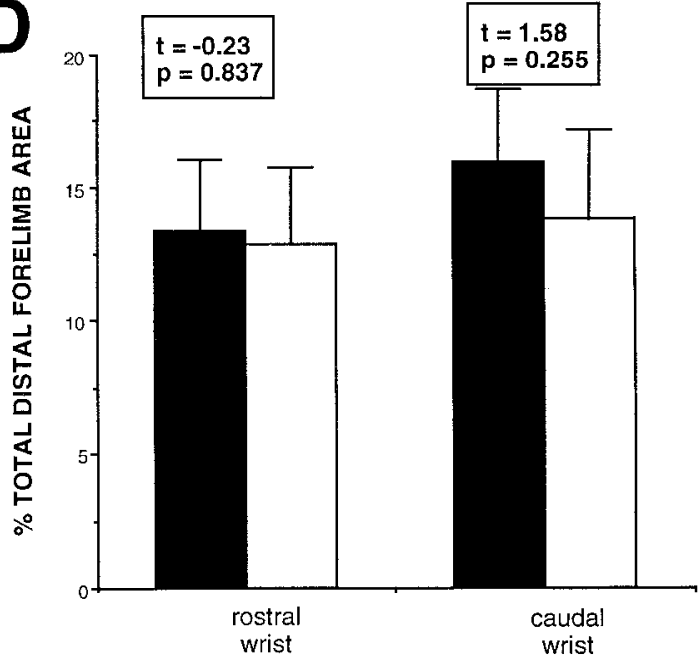

Figure 10. Changes in representation of selected movements in rostral and caudal sectors of distal forelimb representation after digit training. $A$, Percentage of finger (inclusive) area was increased in both rostral and caudal sectors after training, but this increase was somewhat larger (although not statistically significant) in the rostral sector. $B$, Percentage of finger flexion + other area (finger flexion dual-response area) was larger in both rostral and caudal sectors after training, but this increase was significant only in the rostral sector. $f$. Flexion. $C$, Percentage of wrist abduction area was smaller in both rostral and caudal sectors after training, hut was significant only in the rostral sector. $D$, Rostral-caudal differences were not evident in other movement representations, such as percentage of wrist area. Asterisks indicate statistically significant differences $(p<0.05)$. pre, Pre-training; post, post-training.

was identical to phase II training. The animal required $30 \mathrm{~d}$ to reach criterion performance during reacquisition compared with $39 \mathrm{~d}$ during initial training. On the last day of training, the animal's performance was better than at the end of initial training (number of retrievals - 1068; finger flexions per retrieval - 1.33). The next day, a fifth motor-mapping procedure was conducted. Behavioral performance during each of five training phases is summarized in Figure 11.

\section{Movements and movement combinations used in behavioral task}

To assess quantitatively the changes in movements and movement combinations used during each phase of the motor task, frameby-frame analysis of videotapes was done. Samples consisting of selected trials at each of five stages of training on the smallest well (well 4) were examined. These were as follows: (1) baseline, the first 5 trials; (2) phase I training, the first 25 trials on the last day of phase I training; (3) phase II training, the first 25 trials on the last day of phase II training; (4) extinction, 25 probe trials $29 \mathrm{~d}$ after the postextinction map; and (5) reacquisition training, the first 25 trials on the last day of reacquisition training. Note that to minimize the effects of training in the analysis of the baseline behavior, this sample consisted of the first 5 trials, rather than 25 trials in subsequent behavioral samples.

For each stage of training, the frequency of each movement category and movement combination was tallied and expressed as a percentage of total movement events for that stage (Table 3 ). Although this frame-by-frame analysis was useful in describing the frequency of most movement categories, the frequency of thumb movements was not obtained, because the thumb was usually pressed against the apparatus or hidden. 
A

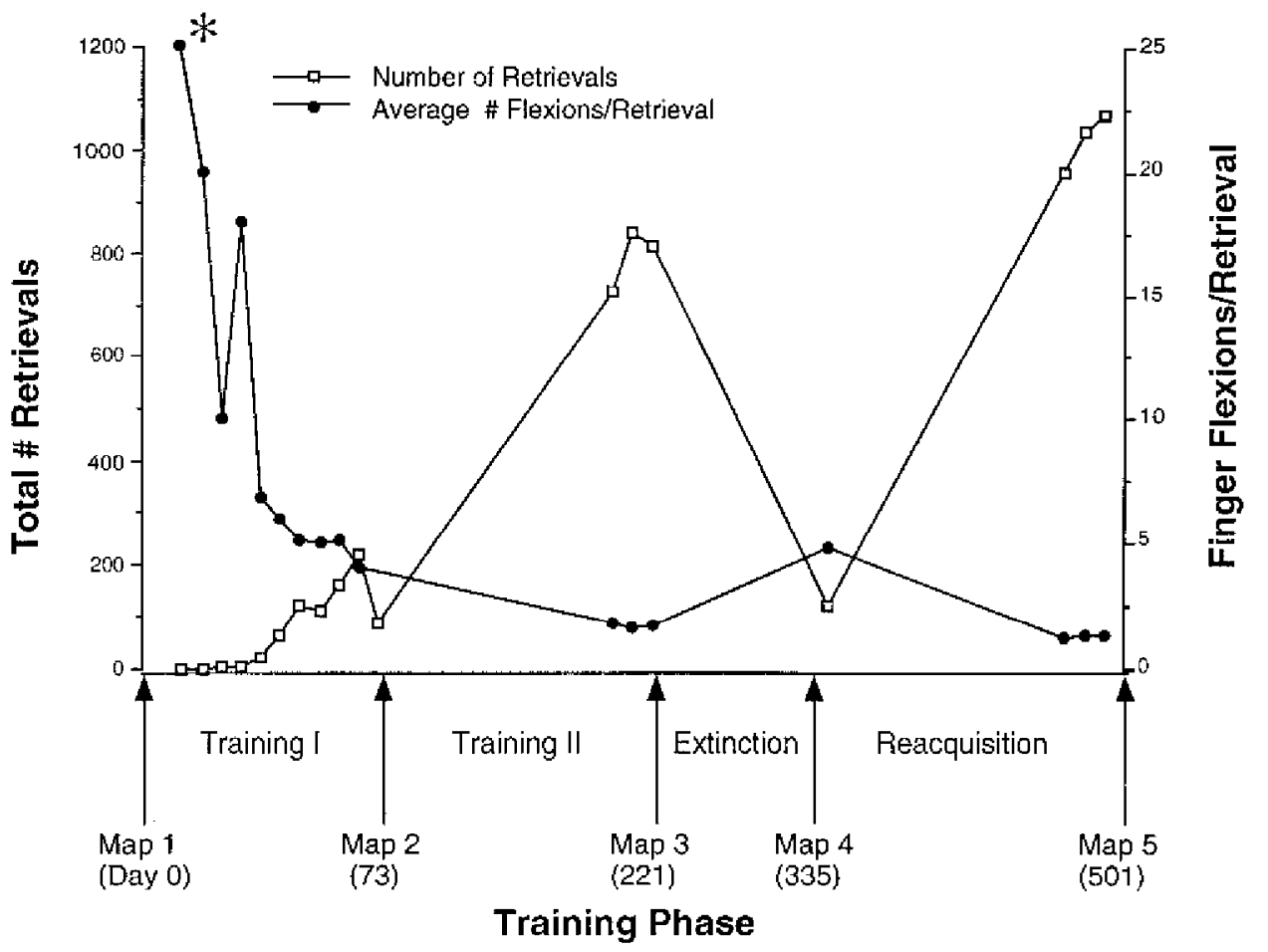

B

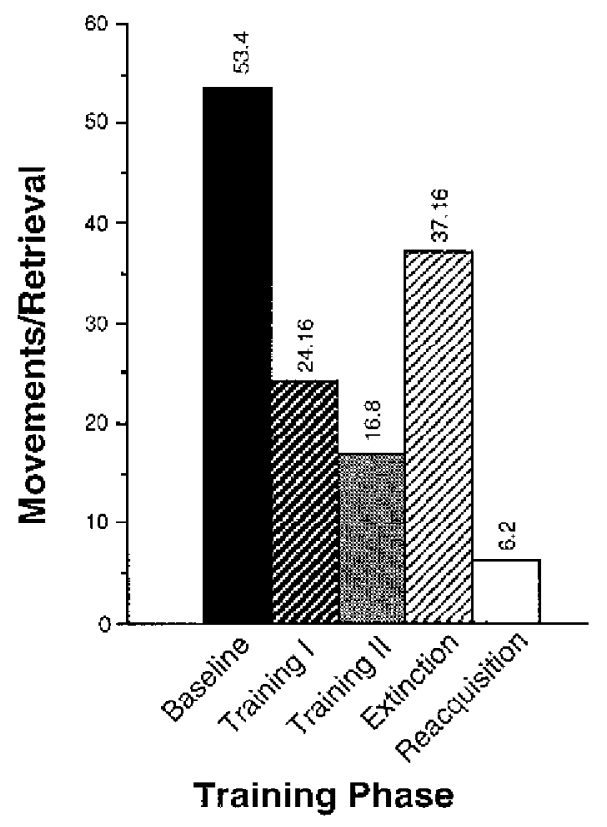

Figure 11. Progression of behavioral performance during five phases of a digit-training task. $A$, Double-Y plot slowing number of relrievals (open squares) and average number of flexions per retrieval (filled circles). Day numbers tefer to days after baseline mapping session (day 0). Timc intervals on the $x$-axis are approximate. Asterisk indicates that no retrievals were obtained after 1200 flexions on the first day of training. $B$, Total number of movements/retrieval based on frame-by-frame analysis of videotapes after each of five phases of training.

The movement analysis showed three trends. First, as training progressed fewer total movements were made per trial (Fig. 11B). Along with the progressive decline in average number of flexions per retrieval (Fig. 11A), these results indicate that the animal's strategy became more efficient with training, involving fewer errots or unsuccessful movements. Second, movement combinations (e.g., finger flexion + wrist extension, etc.) became progressively more frequent. That is, the proportion of movements that comprised movement combinations incrcased with training (Fig. $12 \mathrm{D}$, Table 3). Third, specific movements and movement combinations became more frequent during phases of training in which the behavioral performance was high, that is, at the end of phase If and reacqutisition training (Fig. 12, Table 3). For example, the combination of finger flexion and wrist extension constituted 1.0\% of all distal forclimb movements in the baseline condition, decreased to $0.5 \%$ after phase I training (subcriterion performance), rose substantially to $29.3 \%$ after phasc II training (criterion performance), dropped to $5.0 \%$ after extinction, and rose again to $21.3 \%$ after reacquisition (Fig. $12 F$, Table 3 ). It appeared that this combination was required for successful retrievals, because other movements or movement combinations resulted in a failure to retrieve pellets from well 4 even after phase II or reacquisition training. These results demonstrate that as training proceeded the animal's behavior became more efficient and stereotypic, resulting in a movement strategy that used a limited subset of specific movement combinations to complete the task successfully. 
Table 3. Movements and movement combinations used in behavioral task (case \#1598)

Individual movement categories

\begin{tabular}{lcc}
\hline Movement & Baseline $(\%)$ & Training II $(\%)$ \\
\hline Finger extension & 39.20 & 56.69 \\
Finger flexion & 35.18 & 54.45 \\
Wrist abduction & 4.02 & 1.57 \\
Wrist adduction & 0.50 & 0.00 \\
Forearm supination & 23.12 & 34.55 \\
Forearm pronation & 23.12 & 35.60 \\
Wrist flexion & 0.00 & 1.05 \\
Wrist extension & 9.05 & 34.03 \\
Total & $134.17^{a}$ & 223.56
\end{tabular}

Movement combination categories

\begin{tabular}{lcc}
\hline Movement & Baseline (\%) & Training II (\%) \\
Finger ext./F. pron. & 9.05 & 25.13 \\
Finger ext./F. sup. & 0.00 & 0.52 \\
Finger fl./F. pron. & 1.51 & 0.00 \\
Finger fl./F. sup. & 1.01 & 2.62 \\
Finger fl./Wrist ext. & 1.01 & 29.32 \\
Finger fl./Wrist f. & 0.00 & 0.00 \\
Finger ext./Wrist ext. & 1.01 & 0.52 \\
Finger ext./Wrist fl. & 0.00 & 0.00 \\
Finger fl./Wrist abd. & 0.50 & 0.52 \\
Finger fl./Wrist add. & 0.50 & 0.00 \\
Finger ext./Wrist abd. & 1.01 & 1.05 \\
Finger ext./Wrist add. & 0.00 & 0.00 \\
Wrist ext./F. pron. & 1.01 & 0.00 \\
Wrist fl./F. pron. & 0.00 & 0.00 \\
Wrist ext./F. sup. & 0.50 & 1.57 \\
Wrist fl./F. sup. & 0.00 & 0.00 \\
Total & 17.09 & 61.78
\end{tabular}

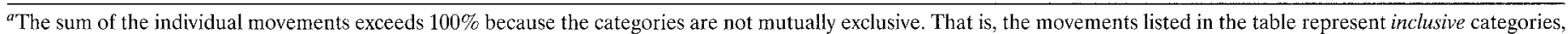
similar to the hierarchical classification for ICMS-evoked movement categories. pron., Pronation; sup., supination. Other abbreviations as in Table 1.

\section{Electrophysiological results}

Analysis of distal forelimb representations in the five maps revealed that changes in the relative areal extent of several of these movement categories were appropriate for each of the five training phases preceding the mapping experiment. For example, the areal extent of the (inclusive) finger area declined slightly from the baseline map to the post-training I map from 40.3 to $34.5 \%$ of the total distal forelimb area. The inclusive finger area then rose to $55.9 \%$ in the post-training II map, declined to $45.2 \%$ in the postextinction map, then rose again to $51.2 \%$ in the post-reacquisition map. Also, the areal extent of several dual-response categories was highest after successful training and rcacquisition (Figs. 12, 13).

In addition, the changes in relative area of several distal forelimb movement categories closely paralleled changes in the animal's behavioral performance. For example, double-Y plots in Figure 12 reveal that the relative areal extent of the finger flexion + wrist extension representation paralleled the number of retrievals obtained during each phase of behavioral training (Fig. 12B). In contrast, the relative wrist/forearm area was inversely related to the number of retrievals (Fig. 12C). Further still, the changes in relative area of other movement categories (each a dual-response category) paralleled changes in the frequency of these same movement combinations used to perform the task (Fig. 12D-F).

\section{Changes in movement representations after forearm training}

To determine whether changes in movement representations occur using a behavioral paradigm encouraging use of a different set of movements, we trained one animal to make repeated, stereotyped supinations and pronations by turning an eyebolt. The training procedure required a gradual shaping of the animal's forelimb movements over a period of 7 weeks as described in Materials and Methods. During supination, the animal also flexed the digits. Initially, the animal ceased manipulating the eyebolt upon delivery of each reward and retrieved the pellet manually from the trough. However, in the final stages of training, the animal invariably used one arm to turn the eyebolt, maintaining constant contact, retrieving pellets from the automatic dispenser either with the opposite hand or with the mouth.

The results of two pretraining baseline maps and one posttraining map are illustrated in Figure 14. Expressed as a percentage of total distal forelimb area, the areal extent of individual movement representations was relatively unchanged in the two baseline maps (see error bars in Fig. 14).

As in digit training, forearm training resulted in considerable changes in distal forelimb movement representations. However, the changes in specific movement categories were markedly different from those recorded after digit training and, in many respects, reflected the differences in movements used to accomplish the task. For example, the forearm-training case showed a decrease in the finger representation from $49 \%$ of the total distal forelimb area in both of the two pretraining maps to $30.9 \%$ in the post-training map. This decrease was attributable primarily to a decrease in the finger extension representation, which decreased from 26.5 and $35.9 \%$ in the two pretraining maps to $18.1 \%$ in the post-training map (Fig. 14).

In the digit-training cases described above, forearm supination remained relatively constant, increasing by 1.4 and $2.0 \%$ of the total distal forelimb area in two cases and decreasing by $3.4 \%$ in one case. In contrast, in the forearm-training case, forearm supination increased from 4.1 and $2.4 \%$ of the total distal forelimb area in pretraining maps, respectively, to $9.3 \%$ in the post-training map. Forearm pronation area remained unchanged, comprising 4.0 and $1.4 \%$ of the total distal forelimb area in pretraining maps and $2.6 \%$ in the post-training map. One other movement category, wrist abduction, displayed a substantial increase in representational area, increasing from 2.8 and $5.2 \%$, respectively, in pretraining maps, to $11.2 \%$ in the post-training map.

To summarize, these two different training paradigms-one encouraging the increased use of the digits, the other encouraging the increased use of the forearm-resulted in very different changes in distal forelimb movement representations. Digit training led to an 
A

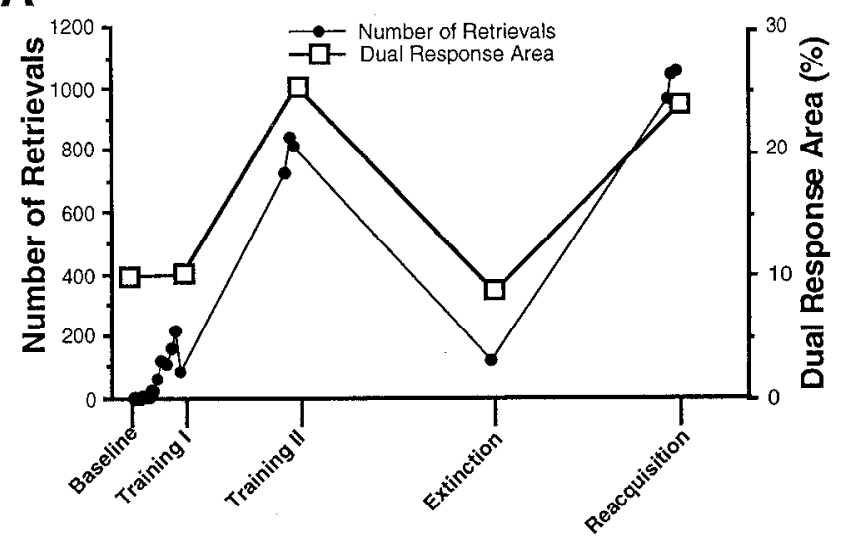

C

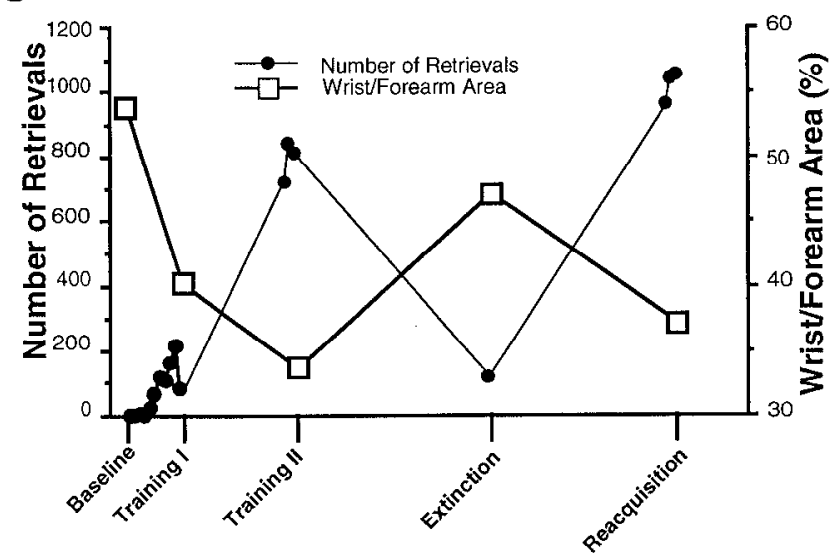

E

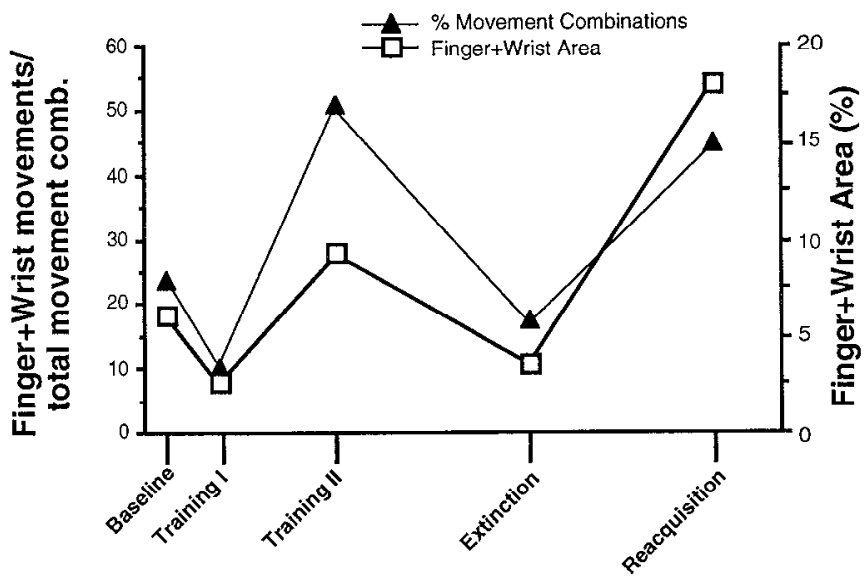

B

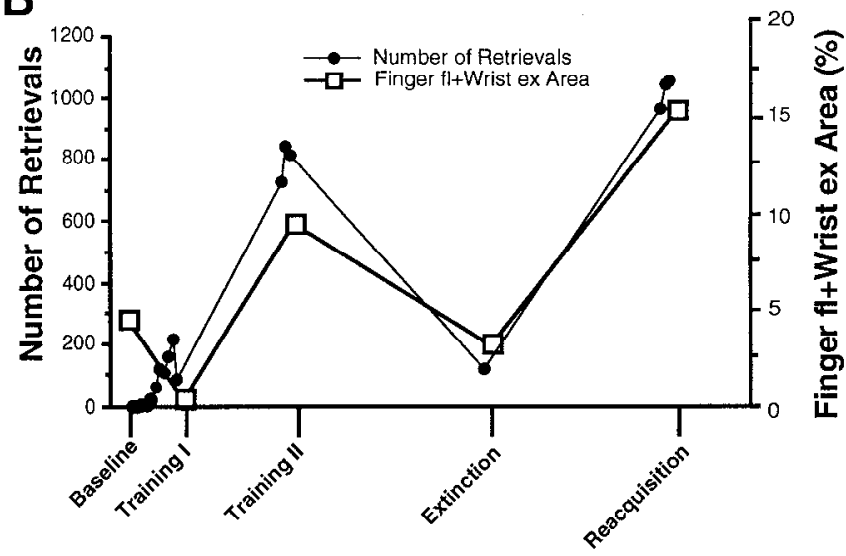

D

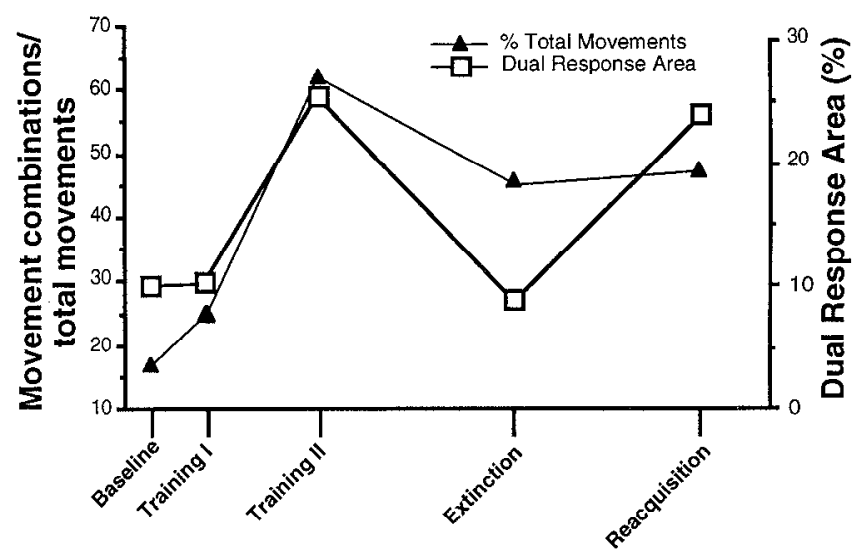

$F$

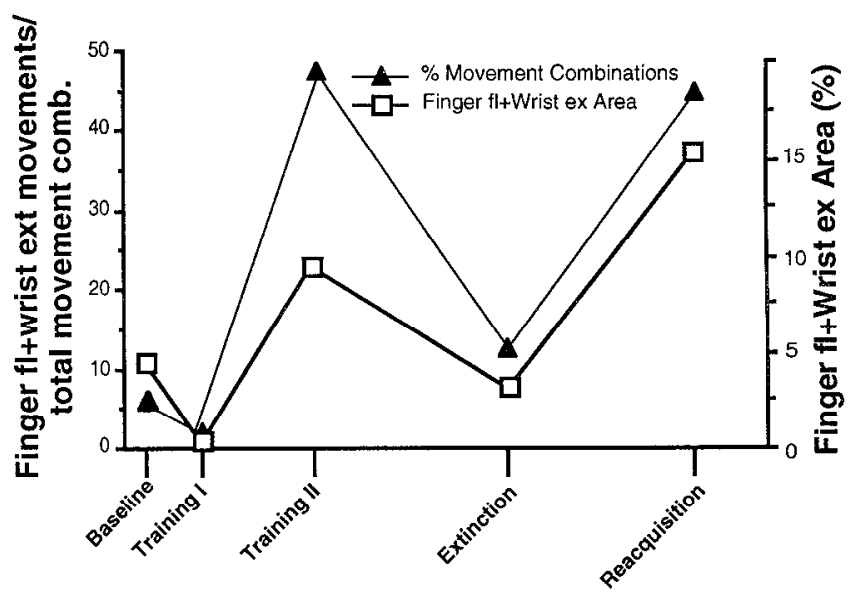

Figure 12. Double-Y plots showing parallel changes in behavioral performance and representational area after each of five phases of a digit-training task (case \#1598). A-C, Behavioral performance is the total number of retrievals for selected training days before each mapping session; representational area is the total cortical area from which dual-responses, finger flexion + wrist extension, and wrist/forearm were evoked. $D$, Behavioral performance is the proportion of total movements comprising movement combinations. Note that this differs from Table 3 , in which movement combinations are expressed as a proportion of total movements. Representational area is the percenlage of distal forelimb area from which dual-responses were evoked. $E$, $F$, Behavioral performance is the proportion of movement combinations that were comprised of finger + wrist and finger flexion + wrist extension, respectively. Representational area is the percentage of distal forelimb area from which these same movements were evoked. See Results for further details. 'I'ime intervals on the $x$-axis are approximate. comb., Combinations; $e x$, extension; $f$, flexion.

increase in representational area devoted to fingers, including finger flexion and extension, and in dual-response areas involving finger movements, especially finger flexion and wrist extension. In addition, digit training resulted in a decrease in the representational area devoted to wrist, especially wrist abduction. By contrast, forearm training resulted in an increase in representational area devoted to wrist abduction and forearm supination and a decrease in representational area devoted to finger extension. 

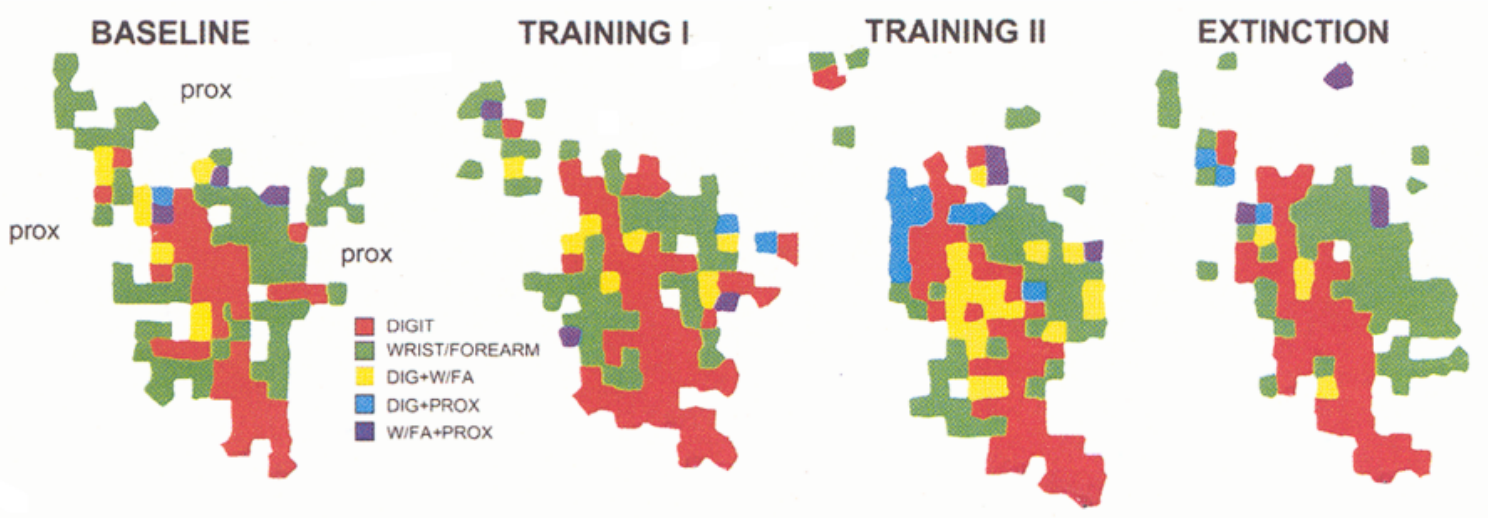

REACQUISITION
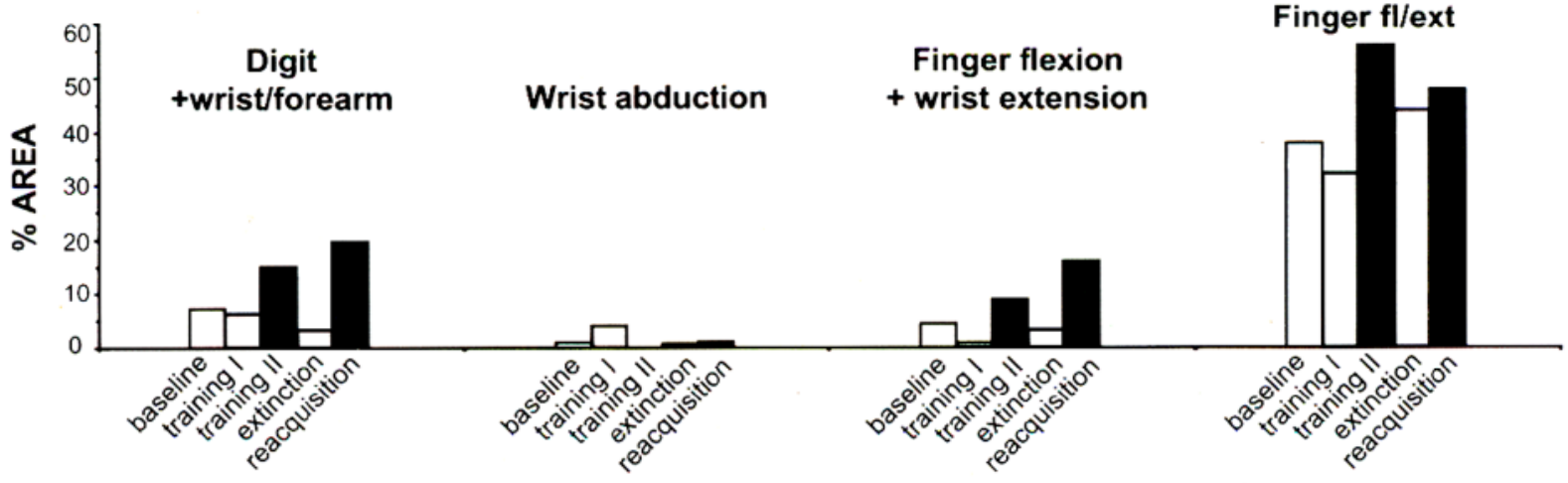

Figure 13. Changes in representation of selected movements after each of five phases of a digit-training task (case \#1598). Phase I training was conducted on a Klüver board for $11 \mathrm{~d}$ and resulted in subcriterion performance. In phase II training ( $39 \mathrm{~d}$ ), we used a titration procedure on the Klüver board, which resulted in criterion performance. During the extinction phase, no behavioral training was conducted for 4 months. The reacquisition phase $(30 \mathrm{~d})$ was identical to phase II training. The areal extent of several movement categories, especially categories consisting of combinations of digit and wrist movements, paralleled the behavioral training procedure. $D I G$, digit; $W / F A$, wrist forearm; $P R O X$, proximal; fl/ext., flexion/extension.

\section{DISCUSSION}

Before discussing the implications and functional significance of these findings, it is important to address possible sources of variability that may have contributed to the alterations in motor map organization recorded in these experiments.

\section{Possible sources of variation in ICMS-defined motor maps}

The significance of the areal changes in movement representations ultimately depends on the reliability of the ICMS technique used to determine the functional organization of M1. The sources of variability in movements evoked by ICMS have been discussed in detail in an earlier paper and by other investigators (Gellhorn and Hyde, 1953; Huang et al., 1988; Nudo et al., 1992; Sanes et al., 1992). However, two aspects of ICMS mapping procedures bear special importance for the present experiments. First, as has been observed in the past, ICMS sometimes evokes movements about multiple joints, even at threshold current levels. Because the interpretation of our results depends in part on the definition of movements evoked at these so-called dual-response sites, careful attention to the potential movement about each joint was required during the ICMS period. We used a standardized criterion for dual-responses: if a second movement was reliably evoked at $\leq 2 \mu \mathrm{A}$ above the threshold intensity, the movements were said to be unresolvable and the site was designated as a dual-response site. The use of a second independent observer further ensured the reliability of these definitions.

Second, although we attempted to control the anesthetic state under ketamine/acepromazine as closely as possible, when neces- sary we temporarily suspended ICMS mapping during periods of deeper or lighter than usual anesthesia. To the extent that variations in anesthetic state may be a significant source of variability in ICMS-defined thresholds, conclusions based on threshold data should be weakened accordingly. Nevertheless, it should be noted that the specific movements evoked by ICMS at a given site did not vary substantially throughout the course of the mapping procedure, despite a slight waxing and waning in anesthetic state.

\section{Stability of ICMS-defined control maps}

It is clear from the present data that ICMS-derived maps of movement representations are somewhat variable from one mapping procedure to the next, even in the absence of specific manipulations. Despite our attempts to control each of the potential sources of variability inherent in ICMS-mapping procedures, movement representations were not identical across time in the same cortical sector of area 4 of the same animal.

Nevertheless, the general topography of specific movement representations was approximately constant in control maps and was not substantially different from that of previously published distal forelimb maps in M1 [Donoghue et al. (1992) and Nudo et al. (1992) in squirrel monkey; Gould et al. (1986) and Stepniewska et al. (1993) in owl monkey; Huntley and Jones (1991) in macaque]. Changes that occurred were small and not consistent from case to case. It is tempting to speculate that even these changes in control cases were attributable to the experimental procedure itself, that is, the direct effects of the surgical intervention, the effects of the microstimulation procedure, and the indirect effects of the surgical intervention on the animal's behavior. 


\section{FOREARM TRAINING CASE 721: SELECTED MOVEMENT REPRESENTATIONS}

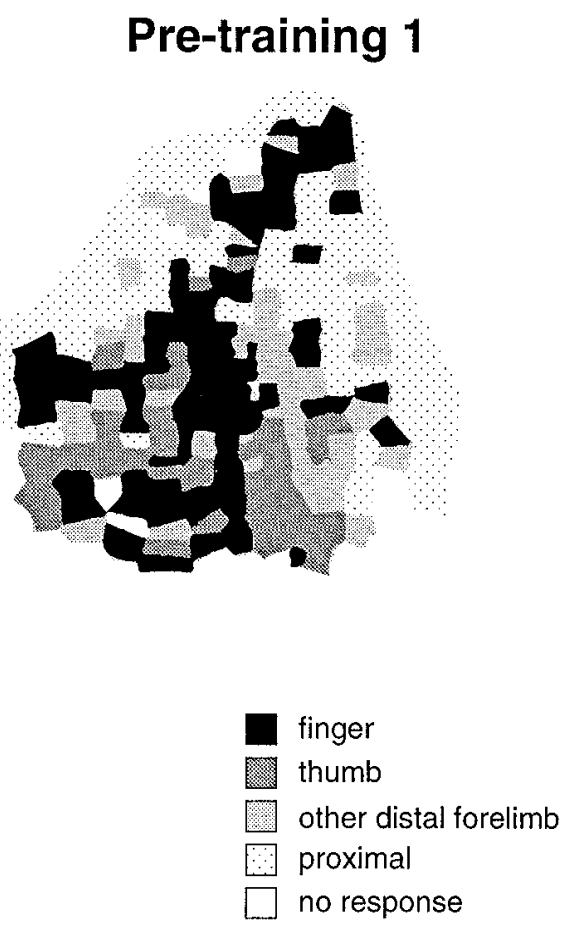

Pre-training 1

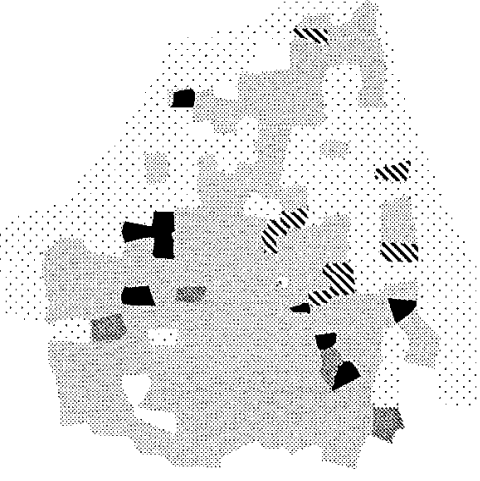

forearm supination

forearm pronation

10. wrist abduction

other distal forelimb

proximal

$\square$ no response
Pre-training 2

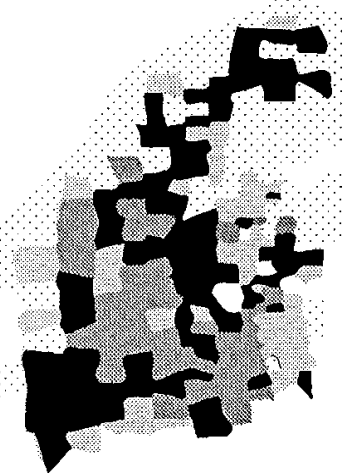

Post-training
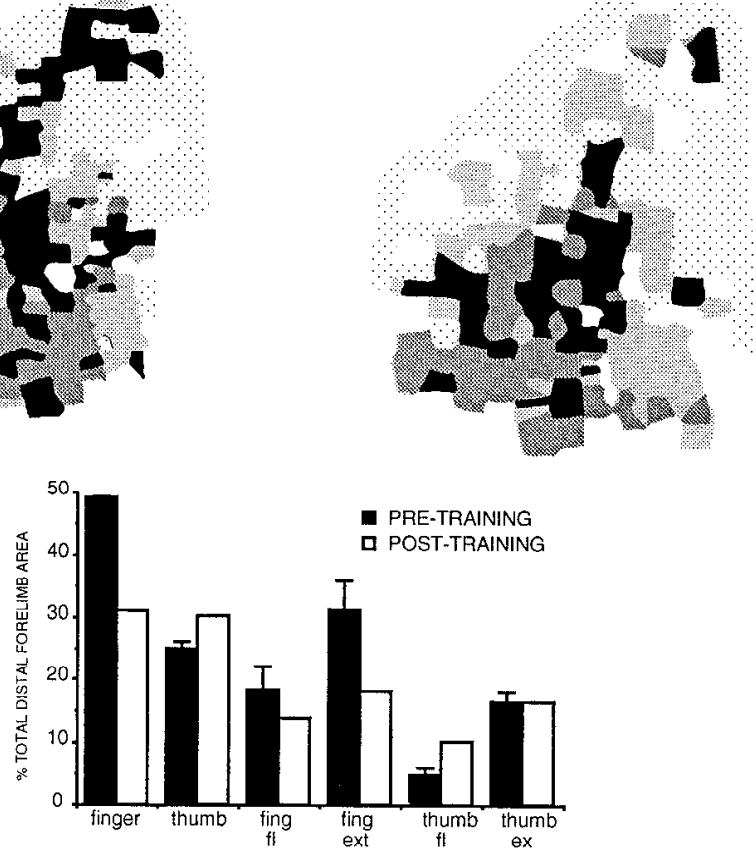

Pre-training 2

Post-training
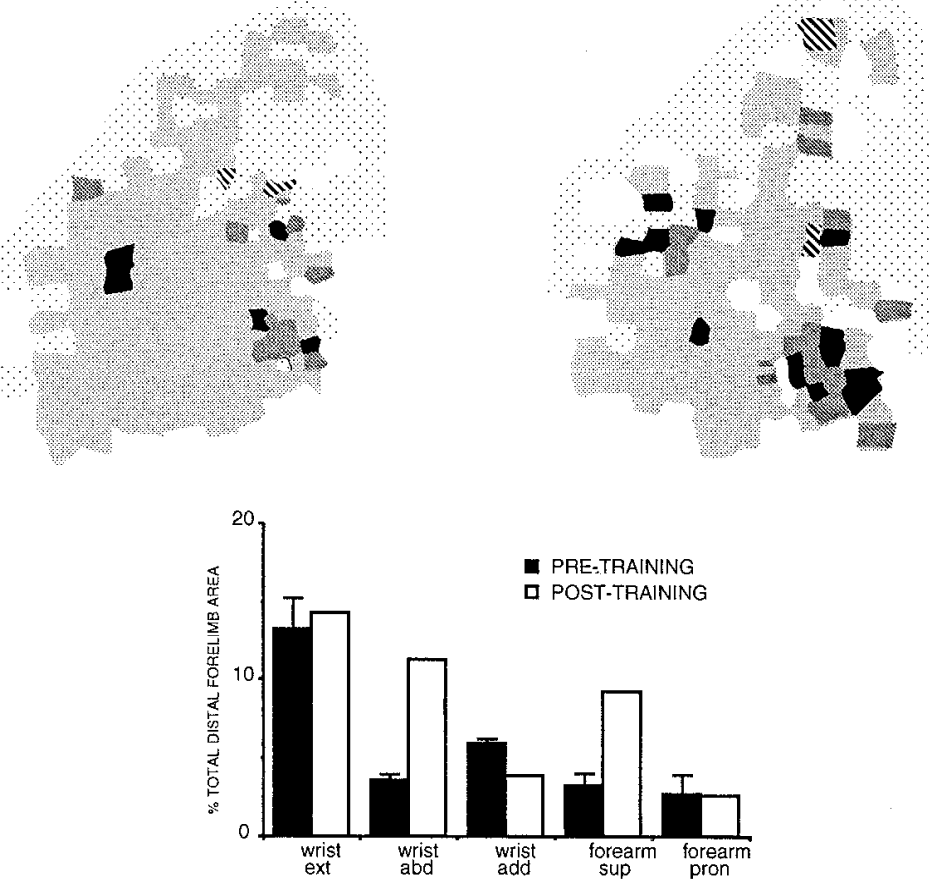

Figure 14. Representation of the distal forelimb in cortical area 4 before and after a training task requiring repetitive forearm supination and pronation. Each of the movement representations depicted in this figure is derived from inclusive categories, that is, they include dual-response sites. Abbreviations as in Tables 1 and 3 . 


\section{Use-dependent reorganization of distal forelimb representations}

In contrast to control cases, the movement representations of monkeys trained on a task requiring skilled movements of the digits changed systematically. The total distal forelimb area did not change significantly, increasing in the three trained animals by an average of $5 \%$. Changes were found primarily in singlemovement digit categories or in dual-response categories involving the combination of a digit movement and another distal forelimb movement (e.g., finger flexion/extension, finger flexion + wrist extension, etc.). These results suggest that as digit skills are acquired very little expansion of the total hand representation occurs. Instead, digit and wrist/forearm movement representations are redistributed within M1. In the present study, finger movement representations (and their combinations) expanded at the expense of wrist representations. In contrast, after training on a task requiring supination and pronation of the forearm, finger movement representations decreased in areal extent, whereas forearm supination and wrist abduction increased. Thus, specific behavioral tasks differentially alter movement representations.

In addition, a repeated mapping experiment derived from five phases of behavioral training suggest that use-dependent changes are progressive and reversible. However, reversibility was not complete after an extinction phase. Although behavioral performance dropped to pretraining levels, finger representations decreased by relatively small amounts (Fig. 12). This discrepancy between behavioral performance and representational area suggests that once a novel motor task is learned certain aspects of functional cortical topography remain altered for an extremely long period of time.

It is interesting to note that the animal in which behavioral performance was initially poor and required a long titration procedure to reach criterion performance on a digit-skill task (case \#1598) also possessed unusually small distal forelimb representations, especially for finger extension. This finding, although anecdotal, suggests that the size of the cortical territory devoted to the hand is directly related to motor skill.

Many of the representational changes could be predicted by behavioral changes associated with the specific motor tasks. However, other changes were expected but not found. First, the lack of a substantial increase in the total distal forelimb area seems contradictory to earlier results. For example, other studies in adult rats demonstrated an expansion of the distal forelimb representation after peripheral nerve lesions (Sanes et al., 1988, 1990; Donoghue et al., 1990), electrical stimulation (Nudo et al., 1990), or passive movements (Humphrey et al., 1990). This apparent discrepancy may be attributable to at least two reasons: (1) in the present study manipulations were strictly behavioral, possibly inducing more subtle changes in cortical topography; (2) the behavioral task required use of muscles throughout the upper extremity, not just the distal forelimb. Thus, changes in elbow and shoulder representations may have counterbalanced, to some extent, expansion of more distal representations.

Second, because the combination of finger flexion and wrist extension seemed to be required for successful pellet retrieval, it was somewhat surprising that the finger flexion area did not change significantly. Significant changes with training occurred only when the finger flexion area was combined with the finger extension area. It is possible that independent finger extension (and finger stabilization) was required for the insertion of specific fingers into the well and that finger flexion + wrist extension was required for retrieval (Fig. 2). Individual differences in the strategies that each animal used may have contributed to variations in finger flexion and finger extension expansion.

Third, after forearm training, the forearm supination representation increased in areal extent but the forearm pronation representation remained constant. No simple explanation can account for this discrepancy, because approximately equal force was required to activate the microswitches during each phase of movement. Future studies using electromyographic analysis of supinators and pronators should reveal whether these muscles are used symmetrically in this task.

Few significant changes in thresholds for activating movements were observed. This result suggests that the increase in areal extent of digit representations seen in this study was not the result of use-dependent changes in excitability of spinal cord motor neurons, as has been hypothesized previously (Leyton and Sherrington, 1917); that is, it is conceivable that a population of corticospinal neurons adjacent to a representational area, say the finger extension area, projects to motor neurons, innervating finger extension (i.e., extensor digitorum) muscles, but activation of these neurons results only in EPSPs in the motor neuron pool. After digit training, it is possible that a use-dependent increase in the excitability of the motor neurons allows activation of the same population of corticospinal neurons to depolarize motor neurons in the pool, resulting in finger movements from a more extensive area of cortcx. Because no systematic decreases in threshold were found, this possibility is unlikely.

Only two significant changes in threshold, both increases, were observed. More current was required to evoke finger movements and finger extension movements after training. This result is important, because it implies that the higher currents required to evoke finger movements after training resulted in an "unmasking" of a preexisting representation. However, finger extension (as well as finger flexion) representations increased in areal extent after training even when movements were defined by using a fixed 20 $\mu \mathrm{A}$ stimulus. Thus, it appears that individual movement representations expand independent of stimulation thresholds.

\section{Development of task-specific combinations of movements (dual-response sites) as a correlate of motor learning}

One of the most consistent results in the present study was that dual-response representations increased in total areal extent after digit training. Although dual-responses were evoked over a relatively small portion of the total distal forelimb area, they were evoked consistently over a more extensive area after training. It is interesting to note that the thresholds for evoking dual-responses are unusually low, especially after training (see Fig. 9)

In addition, examination of videotapes depicting movement strategies used in the acquisition of the digit task revealed that the movement combinations that were used more frequently after training were the same movements that were represented in these expanded dual-response areas. Perhaps more important, movement combinations used in the acquisition of a skilled motor task came to be represented in the same cortical territory. This result suggests that the temporal correlation of movements (and presumably, muscles) drives changes in cortical motor organization. Whether the mechanisms underlying these changes are attributable to temporally correlated outputs from motor cortex or temporally correlated inputs to motor cortex from activated muscles and joints cannot be delineated using the present techniques. However, these results suggest that a neurophysiological correlate 
of a motor skill resides in M1 for at least several days after acquisition. The results also suggest that the combinations of movements evoked by ICMS are attributable partially to the specific temporally correlated movements used by the animal before the ICMS-mapping procedure.

The idea that temporally correlated input drives cortical organization has been suggested in several recent papers in auditory, somatosensory, and visual cortex (Merzenich et al., 1984; Merzenich, 1987; Miller et al., 1989; Singer, 1990; Allard et al., 1991; Recanzone et al., 1992a-c; Dinse et al., 1993). For example, it was shown recently in owl monkey that several weeks to months after suturing two digits so that they received temporally coincident input, double-digit receptive fields were found in primary somatosensory cortex (Allard et al., 1991). The present results are consistent with this hypothesis, because dual-response representations expanded in motor cortex after behavioral training that encouraged coincident movements represented in the expanded territories.

In contrast, some results were not consistent with this hypothesis. For example, after forearm training, changes in dualresponse representations were not observed. Based on the strategy used to turn the key, one would expect an increase in the areal extent of the forearm supination + finger flexion representation. No such increase was found. In fact, the finger flexion area decreased slightly. It is possible that results from the present digit-training task cannot generalize to other tasks. Although unlikely, the emergence of dual-response zones may be correlated uniquely with tasks requiring skilled use of the digits. Also, individual differences in strategies used by different animals may result in very different patterns of reorganization in motor topography.

\section{Rostral-caudal differences in use-dependent reorganization in $\mathbf{M 1}$}

Although changes in movement representations were observed throughout the M1 distal forelimb area, they were larger and more consistent in the rostral half. This result may be important because it has been argued that M1 can be functionally subdivided into rostral and caudal zones based on sensory afferent distributions. Recent anatomical results in owl monkeys suggest that caudal M1 is connected primarily with area 1 of somatosensory cortex (and weakly with area $3 \mathrm{~b}$ ), whereas rostral M1 is connected primarily with area 2 and other motor areas [Stepniewska et al. (1993); see also Jones et al. (1978)]. Electrophysiological results also suggest that rostral and caudal M1 are functionally distinct. Tanji and Wise (1981) found in macaques that cutaneous units were confined primarily to caudal $\mathrm{Ml}$, whereas noncutaneous units predominated in rostral M1. In squirrel monkeys, we have found that although noncutaneous units responding to muscle and joint input are found throughout the M1 hand area, cutaneous units are confined to its most caudal aspect (Humphrey et al., 1994). Finally, Strick and Preston (1982b) also reported that caudal M1 receives primarily cutaneous afferents, whereas rostral M1 receives muscle and joint afferents. However, the relationship between the mosaical representations in M1 found in the present article (but see also Gould et al., 1986; Donoghue et al., 1992; Stepniewska et al., 1993) and the paired digit/wrist representations found by Strick and Preston is unclear. Combined with the present results demonstrating greater changes in representational plasticity in rostral M1, these data suggest that the activation of muscle and joint receptors by specific movements in a motor task plays an important role in the subsequent reorganization of M1.
As a corollary, the activation of culaneous receptors by these same movements plays a less important role in reorganization in $\mathrm{M} 1$, at least in this particular task.

\section{Mechanisms underlying use-dependent reorganization in motor cortex}

Although the mechanisms underlying functional reorganization in motor cortex are difficult to interpret on the basis of techniques used in the present study, experiments in other laboratories have begun to shed light on a possible model. Recently, it has been shown that synaptic efficacy in motor cortex may be modified by the induction of long-term potentiation (LTP), similar to mechanisms that have been studied extensively in hippocampus (Iriki et al., 1989; Keller et al., 1990). Also, local modules within motor cortex are linked via both excitatory and inhibitory intracortical connections (Keller, 1993). In cortical slice preparations in rat motor cortex, LTP can be induced in local intracortical connections, providing a possible mechanism for synaptic plasticity in motor cortex (Keller et al., 1990; Hess and Donoghue, 1994). Application of $N$-methyl-D-aspartate antagonists to motor cortex in rat blocks plasticity induced by passive limb movement (Qiu et al., 1990). Finally, intracellular changes in synaptic effectiveness have been recorded as a consequence of classical conditioning in motor cortex in cat (Woody et al., 1991; Aou et al., 1992). It is possible that changes in synaptic efficacy between neighboring cortical modules underlie the increase in dual-responses seen in the present study. For example, in the baseline condition, ICMS in a particular locus in motor cortex may evoke finger flexion, whereas ICMS in another locus may evoke wrist extension. It is possible that combined use of finger flexion in combination with wrist extension during a motor task enhances intracortical connections between these two modules. Subsequently, ICMS can evoke both digit flexion and wrist extension at the same current level, because excitation of one module then engages the other.

Alternatively, changes in dual-responses evoked by ICMS in M1 may be the result of changes in synaptic efficacy in subcortical brain structures or in the spinal cord. For example, corticospinal neurons are known to branch extensively, often innervating multiple motor neuron pools (Fetz and Cheney, 1980; Shinoda et al., 1981). It is possible that efficacy of selected synapses is altered during motor training. However, according to this model a decrease in thresholds after training would be expected. As described above, decreases in threshold were not recorded.

\section{Functional significance of use-dependent reorganization in motor cortex}

Large changes in topographic organization after acquisition of behavioral tasks have been observed previously in somatosensory, auditory, and visual cortices (Jenkins et al., 1990; Recanzone et al., 1992b,c, 1993). The present experiments extend these findings to M1 (see also Suner et al., 1993). Thus, they represent an important test of the general hypothesis that representational maps are a reflection of individual experiences.

Earlier experiments by Woody and Engel (1972) have demonstrated that large changes in movement representations arise in the motor cortex of Pavlovian-conditioned cals. Specific changes in those studies were related to conditioned responses or to unconditioned stimuli (for review, see Woody, 1986). Fetz and Baker (1972) have shown that isolated cells in motor cortex can be operantly conditioned to increase or decrease their firing rate within minutes. Although these experiments were not designed to provide a reconstruction of changes in the detailed topographies 
of motor representations, they argue strongly that at least the classical conditioning paradigm produces significant changes in distributed neuronal responses in motor cortex.

Recent studies in humans using a variety of techniques have also suggested that primary cortical areas are alterable as a result of motor skill learning. For example, in positron emission tomographic studies, activation of the hand area in M1 was found to increase as subjects practiced sequences of finger movements (Seitz et al., 1990; Grafton et al., 1992; Jenkins et al., 1994; Remy et al., 1994; Schlaug et al., 1994). Using transcranial magnetic stimulation to map the motor cortex, Pascual-Leone et al. (1994) found that improvement in reaction time on a motor task was correlated with enlargement in the output maps of muscles engaged in the task. More recently, using functional magnetic resonance imaging, the extent of motor cortex activated during performance of a motor sequence was found to increase (Karni et al., 1994).

Acquisition and execution of a skilled motor task require the coordinated participation of a number of structures in the motor hierarchy, including motor cortex, basal ganglia, cerebellum, and spinal cord. It is unreasonable to assume that motor cortex is the only locus for use-dependent reorganization in motur systens (Aizawa et al., 1991; Aosaki et al., 1994). It is almost certainly the case that neuronal programs for motor skills are distributed, as has been suggested for storage of memory (Squire, 1992). However, the present results suggest that at least some aspects of acquired motor skills are reflected in the topography of neurophysiological maps in M1.

\section{REFERENCES}

Aizawa H, Inase M, Mushiake H, Shima K, Tanji J (1991) Reorganization of activity in the supplementary motor area associated with motor learning and functional recovery. Exp Brain Res 84:668-671.

Allard T, Clark SA, Jenkins WM, Merzenich MM (1991) Reorganization of somatosensory area $3 \mathrm{~b}$ representations in adult owl monkeys after digital syndactyly. J Neurophysiol 66:1048-1058.

Aosaki T, Tsubokawa H, Ishida A, Watanabe K, Graybiel AM, Kimura M (1994) Responses of tonically active neurons in the primate's striatum undergo systematic changes during behavioral sensorimotor conditioning. J Neurosci 14:3969-3984.

Aou S, Woody CD, Birt D (1992) Increases in excitability of neurons of the motor cortex of cats after rapid acquisition of eye blink conditioning. J Neurosci 12:560-569.

Calford MB, Tweedale R (1988) Immediate and chronic changes in responses of somatosensory cortex in adult flying-fox after digit amputation. Nature 332:446-448.

Calford MB, Tweedale R (1991) Acute changes in cutaneous receptive fields in primary somatosensory cortex after digit denervation in adult flying fox. J Neurophysiol 65:178-187.

Costello MB, Fragaszy DM (1988) Prehension in Cebus and Saimiri. I. Grip type and hand preference. Am J Primatol 15:235-245.

Dinse HR, Recanzone GH, Merzenich MM (1993) Alterations in correlated activity parallel ICMS-induced representational plasticity. NeuroReport 5:173-176.

Donoghue JP, Sanes JN (1987) Peripheral nerve injury in developing rats reorganizes representation pattern in motor cortex. Proc Natl Acad Sci USA 84:1123-1126.

Donoghue JP, Suner S, Sanes JN (1990) Dynamic organization of primary motor cortex output to target muscles in adult rats. II. Rapid reorganization following motor nerve lesions. Exp Brain Res 79:492-503.

Donoghue JP, Leibovic S, Sanes JN (1992) Organization of the forelimb area in squirrel monkey motor cortex: representation of digit, wrist and clbow muscles. Exp Brain Res 89:1-19.

Evarts EV, Fromm C, Kröler J, Jennings VA (1983) Motor cortex control of finely graded forces. J Neurophysiol 49:1199-1215.

Fetz EE, Baker MA (1973) Operantly conditioned patterns of precentral unit activity and correlated responses in adjacent cells and correlated muscles. J Neurophysiol 36:179-204.
Fetz EE, Cheney PD (1980) Postspike facilitation of forelimb muscle activity by primate corticomotoneuronal cells. J Neurophysiol 44:751-772

Gellhorn E, Hyde J (1953) Influence of proprioception on map of cortical responses. J Physiol (Lond) 122:371--385.

Glees P (1961) Experimental neurology. Oxford: Clarendon.

Gould HJ, Cusick CG, Pons TP, Kaas JH (1986) The relationship of corpus callosum connections to electrical stimulation maps of motor, supplementary motor, and the frontal eye fields in owl monkeys. J Comp Neurol 247:297-325.

Grafton ST, Mazziotta JC, Presty S, Friston KJ, Frackowiak RSJ, Phelps ME (1992) Functional anatomy of human procedural learning determined with regional cerebral blood flow and positron emission tomography. J Neurosci 12:2542-2548.

Graham-Brown T, Sherrington CS (1912) On the instability of a cortical point. Proc R Soc Lond [Biol] 85:250-277.

Hess G, Donoghue JP (1994) Long-term potentiation of horizontal connections provides a mechanism to reorganize cortical motor maps. J Neurophysiol 71:2543-2547.

Huang C-S, Sirisko MA, Hiraba H, Murray GM, Sessle BJ (1988) Organization of the primate face motor cortex as revealed by intracortical microstimulation and electrophysiological identification of afferent inputs and corticobulbar projections. J Neurophysiol 59:796-818.

Humphrey DR, Qiu XQ, Clavel P, O'Donoghue DL (1990) Changes in forelimb motor representation in rodent cortex induced by passive movements. Soc Neurosci Abstr 16:422.

Humphrey SM, Gardner GA, Raiszadeh R, Nudo RJ (1994) Loss of sensory, but not motor responsiveness in intact cortex surrounding a focal ischemic infarct in area 4. Soc Neurosci Abstr 20:179.

Huntley GW, Jones EG (1991) Relationship of intrinsic connections to forelimb movement representations in monkey motor cortex: a correlative anatomical and physiological study. J Neurophysiol 66:390-413.

Iriki A, Pavlides C, Keller A, Asanuma H (1989) Long-term potentiation in the motor cortex. Science 245:1385-1387.

Jacobs KM, Donoghue JP (1991) Reshaping the cortical motor map by unmasking latent intracortical connections. Science 251:944-947.

Jenkins IH, Brooks DJ, Nixon PD, Frackowiak SJ, Passingham RE (1994) Motor sequence learning: a study with positron emission tomography. J Neurosci 14:3775-3790.

Jenkins WM, Merzenich MM, Ochs MT, Allard T, Guic-Robles E (1990) Functional reorganization of primary somatosensory cortex in adult owl monkeys after beliaviorally controlled tactile stimulation. J Neurophysiol 63:82-104.

Jones EG, Coulter JD, Hendry SHC (1978) Intracortical connectivity of architectonic fields in the somatic sensory, motor and parietal cortex of monkeys. J Comp Neurol 181:291-348.

Kaas JH (1991) Plasticity of sensory and motor maps in adult mammals. Annu Rev Neurosci 14:137-167.

Kaas JH, Merzenich MM, Killackey HP (1983) The reorganization of somatosensory cortex following peripheral nerve damage in adult and developing mammals. Annu Rev Neurosci 6:325-356.

Kalaska J, Pomeranz B (1979) Chronic paw deafferentation causes an age-dependent appearance of novel responses from forearm in "paw cortex" of kittens and adult cats. J Neurophysiol 42:618-633.

Karni A, Meyer G, Jezzard P, Adams M, Turner R, Ungerleider LG (1994) The acquisition and retention of a motor skill: a functional MRI study of long-term motor cortex plasticity. Soc Neurosci Abstr 20:1291.

Kelehan AM, Doetsch GS (1984) Time-dependent changes in the functional organization of somatosensory cerebral cortex following digit amputation in adult raccoons. Somatosens Mot Res 2:49-81.

Kelehan AM, Ray RH, Carson LV, Massey CE, Doetsch GS (1981) Functional reorganization of adult raccoon somatosensory cerebral cortex following neonatal digit amputation. Brain Res 223:151-159.

Keller A (1993) Intrinsic synaptic organization of the motor cortex. Cereb Cortex 3:430-441.

Keller A, Iriki A, Asanuma H (1990) Identification of neurons producing LTP in the cat motor cortex: intracellular recordings and labeling. J Comp Neurol 300:47-60.

Lawrence DG, Kuypers HGJM (1968) The functional organization of the motor system in the monkey. I. The effects of bilateral pyramidal lesions. Brain 91:1-14.

Leyton ASF, Sherrington CS (1917) Observations on the excitable cortex of the chimpanzee, orangutan, and gorilla. Q J Exp Physiol 11:135-222. 
Merzenich MM (1987) Dynamic neocortical processes and the origirs of higher brain functions. In: The neural and molecular bases of learning (Changeaux JP, Konishi M, eds), pp 337-358. New York: Wiley.

Merzenich MM, Kaas JH, Wall JT, Nelson KJ, Sur M, Felleman D (1983a) Topographic reorganization of somatosensory areas $3 \mathbf{b}$ and 1 in adult monkeys following restricted deafferentation. Neuroscience 8:33-55.

Merzenich MM, Kaas JH, Wall JT, Sur M, Nelson RJ, Felleman DJ (1983b) Progression of change following median nerve section in the cortical representation of the hand in areas $3 b$ and 1 in adult owl and squirrel monkeys. Neuroscience 10:639-665.

Merzenich MM, Nelson RJ, Stryker MP, Cynader MS, Schoppmann A, Zook JM (1984) Somatosensory cortical map changes following digit amputation in adult monkeys. J Comp Neurol 224:591-605.

Merzenich MM, Recanzone G, Jenkins WM, Allard TT, Nudo RJ (1988) Cortical representational plasticity. In: Neurobiology of neocortex (Rakic P, Singer, W, eds), pp 41-67. New York: Wiley.

Miller KD, Keller JB, Stryker MP (1989) Ocular dominance column development: analysis and simulation. Science 245:605-615.

Nudo RJ, Jenkins WM, Merzenich MM (1990) Repetitive microstimulation alters the cortical representation of movements in adult rats. Somatosens Mot Res 7:463-483.

Nudo RJ, Jenkins WM, Merzenich MM, Prejean T, Gedela R (1992) Neurophysiological correlates of hand preference in primary motor cortex of squirrel monkeys. J Neurosci 12:2918-2947.

Pascual-Leone A, Grafman J, Hallett M (1994) Modulation of cortical motor output maps during development of implicit and explicit knowl edge. Science 263:1287-1289.

Qiu XQ, O'Donoghue DL, Humphrey DR (1990) NMDA antagonist (MK-801) blocks plasticity of motor cortex maps induced by passive limb movement. Soc Neurosci Abstr 16:422.

Rasmusson DD (1982) Reorganization of raccoon somatosensory cortex following removal of the fifth digit. J Comp Neurol 205:313-326.

Rasmusson DD, Turnbull BG (1983) Immediate effects of digit amputation on S-I cortex in the raccoon: unmasking of inhibitory fields. Brain Res 288:368-370.

Rasmusson DD, Turnbull BG, Leech CK (1985) Unexpected reorganization of somatosensory cortex in a raccoon with extensive forelimb loss. Neurosci Lett 55:167-172.

Recanzone GH, Allard TT, Jenkins WM, Merzenich MM (1990) Receptive field changes induced by peripheral nerve stimulation in S-I of adult cats. J Neurophysiol 63:1213-1225.

Recanzone GH, Jenkins WM, Hradek GT, Merzenich MM (1992a) Progressive improvement in discriminative abilities in adult owl monkeys performing a tactile frequency discrimination task. J Neurophysiol $67: 1015-1030$

Recanzone GH, Merzenich MM, Jenkins WM (1992b) Frequency discrimination training engaging a restricted skin surface results in an emergence of a cutaneous response zone in cortical area 3a. J Neurophysiol 67:1057-1070.

Recanzone GH, Merzenich MM, Jenkins WM, Grajski KA, Dinse HR (1992c) Topographic reorganizàtion of the hand representation in cortical area $3 \mathrm{~b}$ of owl monkeys trained in a frequency discrimination task. J Neurophysiol 67:1031-1056.

Recanzone GH, Schreiner GE, Merzenich MM (1993) Plasticity in the frequency representation of primary auditory cortex following discrimination training in adult owl monkeys. J Neurophysiol 13:87-103.

Remy P, Zilbovicius M, Leroy-Willig A, Syrota A, Sanison Y (1994) Movement- and task-related activations of motor cortical areas: a positron emission tomographic study. Ann Neurol 36:19-26.

Sanes JN, Suner S, Donoghue JP (1990) Dynamic organization of primary motor cortex output to target muscles in adult rats. I. Long-term palterns of reorganization following motor or mixed peripheral nerve lesions. Exp Brain Res 79:479-491.

Sanes JN, Suner S, Lando JF, Donoghue JP (1988) Rapid reorganization of adult rat motor cortex somatic representation patterns after motor nerve injury. Proc Natl Acad Sci USA 85:2003-2007.

Sanes JN, Wang J, Donoghue JP (1992) Immediate and delayed changes of rat motor cortical output representation with new forelimb configurations. Cereb Cortex 2:141-152.

Schlaug G, Knorr U, Seitz RJ (1994) Inter-subject variability of cerebral activations in acquiring a motor skill: a study with positron emission tomography. Exp Brain Res 98:523-534.

Seitz RJ, Roland RE, Bohm C, Greitz T, Stone-Elander S (1990) Motor learning in man: a positron emission tomographic study. NeuroReport $1: 57-60$.

Shinoda Y, Yokota J-I, Futami T (1981) Divergent projection of individual corticospinal axons to motoneurons of multiple muscles in the monkey. Neurosei Lett 23:7-12.

Simons DJ, Durham D, Woolsey TA (1984) Functional organization of mouse and rat $\mathrm{SmI}$ barrel cortex following vibrissal damage on different postnatal days. Somatosens Mot Res 1:207-245.

Singer W (1990) Search for coherence: a basic principle of cortical selforganization. Concepts Neurosci 1:1-26.

Squire LR (1992) Memory and the hippocampus: a synthesis from findings with rats, monkeys and humans. Psychol Rev 99:195-231.

Stepniewska I, Preuss TM, Kaas JH (1993) Architectonics, somatotopic organization, and ipsilateral cortical connections of the primary motor area (M1) of owl monkeys. J Comp Neurol 330:238-271.

Strick PL, Preston JB (1982a) Two representations of the hand in area 4 of a primate. I. Motor output organization. J Neurophysiol 48:139-149.

Strick PL, Preston JB (1982b) Two representations of the hand in area 4 of a primate. II. Somatosensory input organization. J Neurophysiöl $48: 150-182$.

Suner S, Gutman D, Sanes JN, Donoghue JP (1993) Reorganization of monkey motor cortex related to motor skill learning. Soc Neurosci Abstr 19:775

Tanji J, Wise SP (1981) Submodality distribution in sensorimotor cortex of the unanesthetized monkey. J Neurophysiol 45:467 481 .

Wall JT (1988) Development and maintenance of somatotopic maps of the skin: a mosaic hypothesis based on peripheral and central contiguities. Brain Behav Evol 31:252-268.

Wall JT, Kaas JH (1986) Long-term cortical consequences of reinnervation errors after nerve regeneration in monkeys. Brain Res 372:400-404.

Welker WI, Soriano E, Van der Loos H (1989) Plasticity in the barrel cortex of the adult mouse: effects of peripheral deprivation on $G \wedge D$ immunoreactivity. Exp Brain Res 74:441-452.

Woody CD (1986) Understanding the cellular basis of memory and learning. Annu Rev Psychol 37:433-493.

Woody CD, Engel Jr J (1972) Changes in unit activity and thresholds to electrical microstimulation at coronal-pericruciate cortex of cat with classical conditioning of different facial movements. J Neurophysiol 35:230-241.

Woody CD, Gruen E, Birt D (1991) Changes in membrane currents during Pavlovian conditioning of single cortical neurons. Brain Res 539:76-84.

Zar JH (1984) Biostatistical analysis, 2nd Ed. Englewood Cliffs, NJ: Prentice-Hall. 